\title{
Numerical Investigation of the Effects of Continuum Breakdown on Hypersonic Vehicle Surface Properties
}

\author{
Timothy D. Holman* and Iain D. Boyd ${ }^{\dagger}$ \\ Department of Aerospace Engineering, University of Michigan, Ann Arbor, MI, 48109
}

\begin{abstract}
A hypersonic vehicle crosses many regimes from rarefied to continuum due to the change in density with altitude during the course of its trajectory through a planet's atmosphere. This variation makes it difficult to simulate the flow since the physical accuracy of computational fluid dynamics (CFD) can break down in rarefied flows and the direct simulation Monte Carlo (DSMC) method is computationally expensive in continuum flows. This study investigates the effects of continuum break down on the surface aerothermodynamic properties (pressure, shear, heat transfer rate) of a sphere in Mach 10, 25, and 45 flow of nitrogen gas in regimes varying from continuum flow to rarefied gas flow. A rotational energy relaxation model is employed in the CFD code and is tested to confirm its accuracy. As the global Knudsen number is increased, from continuum flow to a rarefied gas, the amount of continuum breakdown seen in the flow and on the surface is increased. This increase in continuum breakdown affects the surface properties, such that an increase in the differences between CFD and DSMC is observed. As the Mach number is increased, the amount of continuum breakdown observed in the flow is increased, but the gradient length local Knudsen number stays approximately constant. Even though the amount of continuum breakdown has increased, the difference between CFD and DSMC remains relatively constant. The last part of this study compares the results of the sphere with that of the analogous cylinder case. At the same global Knudsen number, the differences in the surface properties between CFD and DSMC increase when the simulation is run axisymmetrically.
\end{abstract}

\section{Nomenclature}

$\begin{array}{ll}R e & \text { Reynolds Number } \\ K n_{\infty} & \text { Global Knudsen Number } \\ K n_{G L L} & \text { Gradient Length Local Knudsen Number } \\ L & \text { Characteristic Length, } \mathrm{m} \\ U & \text { Free Stream Velocity, } \mathrm{m} / \mathrm{s} \\ q & \text { Heat Transfer Rate, } \mathrm{W} / \mathrm{m}^{2} \\ P & \text { Pressure, Pa } \\ T & \text { Translational Temperature, } \mathrm{K} \\ \mu & \text { Viscosity, Pa.s } \\ \tau & \text { Shear Stress, Pa } \\ \rho & \text { Density, } \mathrm{kg} / \mathrm{m}^{3} \\ \lambda & \text { Mean Free Path, } \mathrm{m}\end{array}$

\section{Introduction}

With a renewed desire to send humans back to the Moon and beyond there is a need for accurate studies of the flow behavior over hypersonic vehicles to precisely determine how they will perform when entering

\footnotetext{
${ }^{*}$ Graduate Student, Member AIAA.

$\dagger$ Professor, Associate Fellow AIAA.
} 
an atmosphere. Experiments and flight tests are extremely difficult and expensive, so there is need for a computational models that can be utilized for design and development of hypersonic vehicles.

A hypersonic vehicle that is entering the Earth's atmosphere will go through many different flow regimes due to the change in atmospheric density with altitude. The flow can be characterized by the Reynolds number (Re) and the Knudsen number (Kn) as given in Eq. (1).

$$
R e=\frac{\rho U L}{\mu} \quad K n=\frac{\lambda}{L} \propto \frac{1}{\rho L}
$$

where $\rho$ is the density, $\mu$ is the viscosity, $\lambda$ is the mean free path, $\mathrm{U}$ is the velocity and $\mathrm{L}$ is the characteristic length scale. The flow can be considered a continuum when the Knudsen number is much less than one. In the continuum regime, flows should be simulated using traditional computational fluid dynamics techniques by numerically solving the Navier-Stokes equations. However, when the Knudsen number becomes larger the continuum assumption in the Navier-Stokes equations starts to break down. This is due to the fact that these equations are derived from kinetic theory based on the assumption of small perturbations from an equilibrium velocity distribution function $;^{1}$ therefore CFD only works in near equilibrium flows. When the Knudsen number grows, only a non-continuum technique can be used, such as the direct simulation Monte Carlo method. ${ }^{2}$ DSMC is a Monte Carlo particle method for simulating nonequilibrium gas flows. DSMC is required for accurate flow analysis of hypersonic rarefied flows where the continuum flow equations are invalid, and can be utilized in any dilute gas flow. Unfortunately, DSMC is about an order of magnitude more expensive than traditional CFD methods and becomes prohibitively expensive at low Knudsen numbers. Note that even if the global flow behaves as a continuum there can still be parts of the flow that locally act as a rarefied flow, if the local length scale is very small or the local density is low. For example a hypersonic blunt body can create a locally rarefied flow in the shock, the boundary layer and the wake of the body. As a result, neither CFD nor DSMC can provide a complete computational model across all regimes of a hypersonic vehicle.

One solution to this problem is a hybrid code that utilizes CFD and DSMC methods to accurately and efficiently simulate a hypersonic flow. This hybrid code can solve the Navier-Stokes equations when the flow is considered to be a continuum, but it can switch to a DSMC method when the flow is considered rarefied such as in a shock wave. This process involves some way of finding when the physics of the simulation provided by the CFD method deviates from physical results; this is done with the continuum breakdown parameter. This continuum breakdown parameter will notify the code that the CFD method can no longer be employed and that DSMC needs to be utilized to correctly simulate the flow field. Boyd, et al. ${ }^{3}$ suggested the use of the maximum gradient length local Knudsen number as a continuum breakdown parameter given in Eq. (2).

$$
K n_{G L L}=\frac{\lambda}{Q}\left|\frac{d Q}{d l}\right|
$$

where the derivative is taken in the direction of maximum gradient, and $\mathrm{Q}$ is a variable of interest such as density, temperature or pressure. It has been found that a value of $K n_{G L L}$ above 0.05 indicates continuum breakdown has occurred.

To be able to design a hypersonic vehicle it is important to understand how the continuum breakdown affects the surface conditions such as heat flux, pressure and shear stress. These surface conditions determine the aerodynamic and thermodynamic performance of a re-entry vehicle. A previous study by Lofthouse et al. looked at the effect of break down on the surface properties of a 12 inch diameter two dimensional cylinder in a Mach 10 flow of argon. ${ }^{4}$ A more recent study by Lofthouse et al. examines the effects of velocity slip and temperature jump at the surface of a two dimensional 12 inch diameter cylinder in Mach 10 and 25 flow of argon. ${ }^{5}$ Both of these studies were conducted over a range of Knudsen numbers, 0.002 to 0.25 , where the characteristic length is taken to be the diameter of the cylinder. The present study will continue to examine the continuum break down phenomenon in Mach 10, 25 and 45 flow of nitrogen over a 12 inch sphere with the same global Knudsen number range as the previous investigations. This gives a range of flow conditions from continuum to rarefied gas. The purpose of this work is to accurately characterize the effects of continuum breakdown. This has to be done by starting out with simple simulations and then adding complexity to determine individual effects on continuum breakdown. The work done by Lofthouse et al. started this effort by characterizing breakdown over a two dimensional cylinder in a flow of argon. This study continues to characterize breakdown by adding the complexity of vehicles more representative of real life such as capsules 
in a flow more representative of a real gas such as air. While this study is concerned with nitrogen it does not include chemical reactions, that will be included in future studies.

This paper will discuss the simulation procedures as well as the computational models used to run the simulations. Selected results are presented in four parts. First, the results of the rotational energy model in CFD are given to determine the accuracy of the model. Second, the results of the simulations from the whole range of global Knudsen numbers at a Mach number of 10 are discussed. Third, the results from the simulations of a global Knudsen number of 0.01 at all three Mach numbers are presented. Fourth, a comparison is made of the results from the sphere and cylinder simulations. Finally, conclusions and future work are discussed.

\section{Background}

This study examines the continuum break down phenomenon in flows of nitrogen over a 12 inch diameter sphere at Mach numbers of 10, 25 and 45. The free stream temperature is $200 \mathrm{~K}$ giving free stream velocities of $2883.5 \mathrm{~m} / \mathrm{s}, 7208.75 \mathrm{~m} / \mathrm{s}$ and $12975.75 \mathrm{~m} / \mathrm{s}$ for the three different Mach numbers. The surface of the sphere has fixed temperatures of $500 \mathrm{~K}, 1500 \mathrm{~K}$ and $2500 \mathrm{~K}$ for the Mach 10,25 and 45 cases, respectively. The density of the free stream is varied to change the global Knudsen number of the flow as given in Table 1 . The Knudsen number is calculated using the sphere diameter as the characteristic length and the hard sphere model to calculate the mean free path. From Table 1 it is seen that the global Knudsen number varies from 0.002 to 0.25 ; giving a range of flow regimes from continuum to rarefied gas flow. The simulations are performed using two different computational methods, CFD and DSMC.

Table 1. Simulated flow regimes

\begin{tabular}{|l|l|l|l|}
\hline$K n_{\infty}$ & $\begin{array}{l}\text { Mass Density } \\
\left(\mathrm{kg} / \mathrm{m}^{3}\right)\end{array}$ & $\begin{array}{l}\text { Number Density } \\
\left(\text { particles } / \mathrm{m}^{3}\right)\end{array}$ & $\begin{array}{l}\text { Mean Free Path } \\
(\mathrm{m})\end{array}$ \\
\hline \hline 0.002 & $9.875 \times 10^{-5}$ & $2.124 \times 10^{21}$ & $6.096 \times 10^{-4}$ \\
0.01 & $1.975 \times 10^{-5}$ & $4.247 \times 10^{20}$ & $3.048 \times 10^{-3}$ \\
0.05 & $3.949 \times 10^{-6}$ & $8.494 \times 10^{19}$ & $1.524 \times 10^{-2}$ \\
0.25 & $7.899 \times 10^{-7}$ & $1.699 \times 10^{19}$ & $7.62 \times 10^{-2}$ \\
\hline
\end{tabular}

\section{Numerical Methods}

The DSMC simulations are performed using MONACO ${ }^{6}$ a code developed at University of Michigan by Boyd et al. MONACO utilizes DSMC to accurately simulate rarefied gas flows around hypersonic vehicles. MONACO is a parallel, unstructured, 2D/3D DSMC code, and it includes variable vibrational and rotational energy exchange models. MONACO can use either the variable hard sphere(VHS) or variable soft sphere(VSS) collision models; ${ }^{2}$ the VHS model is employed in this study. The mesh used for the final simulation is adapted by hand from previous simulations such that the cell size is of the same order as the mean free path. For this study a hybrid mesh is utilized with cell stretching. A hybrid mesh is one where both a structured and unstructured grid is utilized. In this case a structured grid is employed along the fore body surface while an unstructured mesh is used everywhere else in the flow field as shown in Fig. 1. For the structured grid, cell stretching is employed. This means the cell widths are adapted to be on the order of a mean free path while the cell heights are stretched larger than the mean free path. The amount of cell stretching varies depending on the global Knudsen number. The grid given in Fig. 1 has cells stretched by a factor of 8 near the stagnation point. The amount of cell stretching decreases farther away from the stagnation point, and eventually the cells are no longer stretched. This procedure creates a larger cell volume so more particles can populate the cells near the stagnation point. This is important in axisymetric simulations where it is difficult to obtain an appropriate number of particles per cell. ${ }^{7}$ This does not affect the simulation results because the primary flow gradients along the stagnation line are aligned with the cell widths, which are small enough to properly simulate the flow. Figure 1(d) shows the unstructured mesh on top of a structured mesh near the stagnation point. From this figure it can be seen that the stretched cells give a much larger area than unstructured cells thus allowing more particles to populate the area near the 
stagnation point.

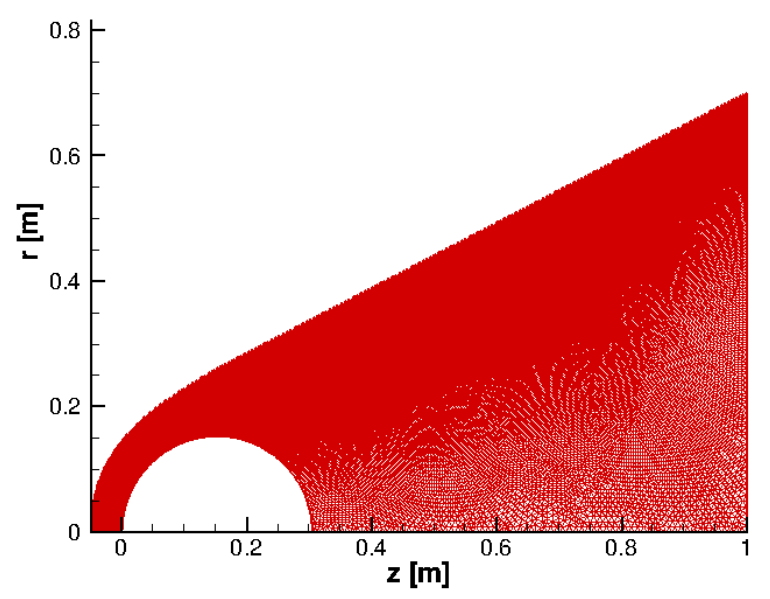

(a) Full Grid

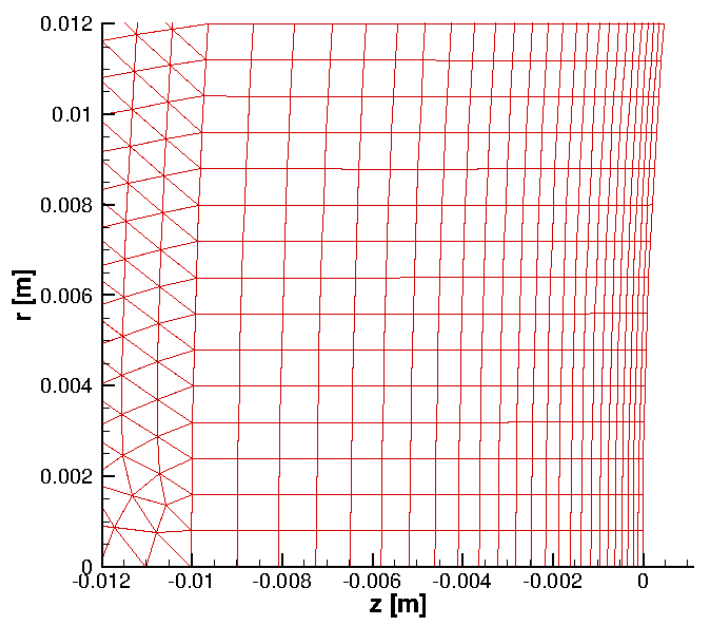

(c) Stagnation Point

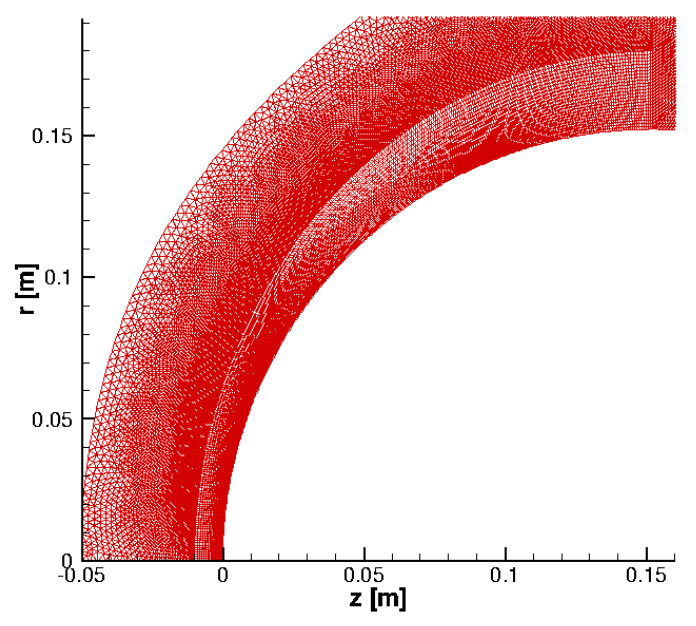

(b) Fore Body

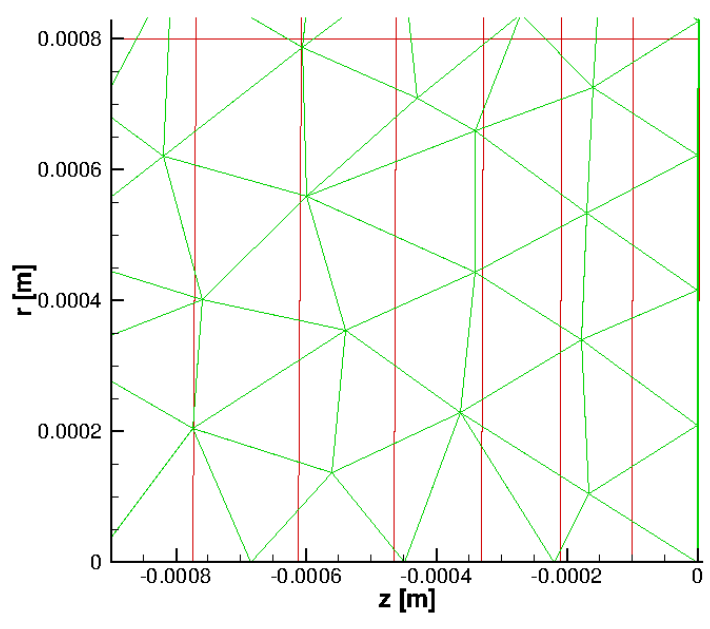

(d) Hybrid and Unstructured Mesh

Figure 1. The final tailored grid for the Mach 10 global Knudsen number 0.01 case for DSMC, all other DSMC grids are similar

The CFD simulations are performed by solving the Navier-Stokes equations by use of the Michigan aerothermodynamics Navier-Stokes code LeMANS, developed at the University of Michigan for the simulation of hypersonic reacting flows. ${ }^{8,9}$ LeMANS is a parallel, unstructured $2 \mathrm{D} / 3 \mathrm{D}$, finite-volume CFD code. LeMANS has the ability to simulate gases in chemical and thermal nonequilibrium. LeMANS employs a modified Steger-Warming Flux Vector Splitting to discretize the numerical fluxes between cells, which has low dissipation and is appropriate near boundary layers. A point implicit method is employed for the time march, but after a few hundred iterations is switched to line implicit method for faster convergence. Even though LeMANS can handle unstructured meshes, all the simulations performed for this study are carried out using structured meshes. It is necessary to ensure that the transport properties are the same with both CFD and DSMC; therefore LeMANS is modified to use the same viscosity as the VHS method as given in Eqs. (3) and (4). ${ }^{10}$

$$
\mu=\mu_{\text {ref }}\left(\frac{T}{T_{\text {ref }}}\right)^{\omega}
$$




$$
\mu_{r e f}=\frac{15 \sqrt{\pi m k T_{r e f}}}{2 \pi d_{r e f}^{2}(5-2 \omega)(7-2 \omega)}
$$

where the variable hard sphere parameters are those used in the DSMC simulations, $\omega$ is 0.75 with a reference diameter and temperature of $4.17 \times 10^{-10} \mathrm{~m}$ and $273 \mathrm{~K}$, respectively.

Even though LeMANS is capable of modeling thermal nonequilibrium it does so with a two temperature model involving a coupled translational-rotational temperature and a coupled vibrational-electronic temperature. DSMC employs a variable rotational energy exchange probability ${ }^{11}$ and therefore simulates has a separate rotational temperature. Since this study is concerned with comparisons between DSMC and CFD for simulations that involve significant amounts of nonequilibrium flow, it is important that the CFD code also has a separate rotational temperature to be able to match DSMC. Not only is it important to have rotational nonequilibrium for comparisons between CFD and DSMC, it is also beneficial to have a separate rotational energy equation that can provide more accurate results in hypersonic flows where thermal nonequilibrium is common. The rotational energy equation per unit volume is given by Eq. (5).

$$
\frac{\partial E_{r}}{\partial t}+\nabla \cdot\left(E_{r} \vec{u}\right)=-\nabla \cdot\left(\overrightarrow{q_{r}}\right)-\nabla \cdot \sum_{s}\left(\rho_{s} e_{r s} \overrightarrow{u d s}_{d s}\right)+\dot{w}_{r}
$$

where $q_{r}$ is the rotational heat flux given by Fourier's law, $u_{d s}$ is the species diffusion given by Fick's law and $\dot{w}_{r}$ is a source term given by Eq. (6).

$$
\dot{w}_{r}=\sum_{s}\left(Q_{r s}^{t-r}+\dot{w}_{s} e_{r s}\right)
$$

Equation (6) is composed of two parts, the first being the rotational energy relaxation given in Eq. (7) and the second part is the gain and loss of rotational energy due to chemical reactions.

$$
Q_{r s}^{t-r}=\rho_{s} \frac{e_{r s}^{*}-e_{r s}}{Z_{r s} \tau_{c}}
$$

The rotational energy relaxation is modeled using a Landau-Teller model, where $e_{r s}^{*}$ is the rotational energy evaluated at the translational temperature, $Z_{r s}$ and $\tau_{c}$ together give the rotational relaxation time. $Z_{r s}$ is the rotational collision number given in Eq. (8) derived by Parker. ${ }^{12} \tau_{c}$ is the mean collision time from the VHS model. Equation (9) gives the mean collision rate, given by Bird, ${ }^{2}$ which is just the inverse of the mean collision time,

$$
\begin{gathered}
Z_{r s}=\frac{Z_{r s}^{\infty}}{1+\frac{\pi^{\frac{3}{2}}}{2}\left(\frac{T_{s}^{*}}{T}\right)^{\frac{1}{2}}+\left(\frac{\pi^{2}}{4}+\pi\right)\left(\frac{T_{s}^{*}}{T}\right)} \\
\nu_{s}=\sum_{i}\left(n_{i}\left(d_{r e f}\right)_{i}^{2}\left(\frac{8 \pi k\left(T_{r e f}\right)_{i}}{m_{i}^{*}}\right)^{\frac{1}{2}}\left(\frac{T}{\left(T_{r e f}\right)_{i}}\right)^{1-\omega_{i}}\right)
\end{gathered}
$$

where $Z_{r s}^{\infty}$ and $T^{*}$ are constants for a given species, and the VHS parameters are the same ones used in DSMC and the CFD viscosity model.

\section{Results}

The purpose of this study is to compare surface properties predicted by DSMC and CFD simulations, heat flux, pressure and shear stress, to see if any differences occur due to continuum breakdown. Additionally, the integrated drag and the maximum heat flux are also compared from DSMC and CFD. The maximum heat flux is found by averaging the heat flux over the surface of the sphere within the first degree of the stagnation point. This is done to be able to determine the maximum heat flux predicted by DSMC which can have some random noise in the results. For CFD it will be seen that the maximum heat flux sometimes occurs slightly off the stagnation point. Since the CFD solutions are smooth there is no need for averaging and the maximum heat flux is found by finding the maximum value of the heat flux on the surface of the sphere. 
The breakdown parameter is computed from both DSMC and CFD simulation results using Eq. (2), where the derivative is taken in the direction of the maximum gradient. It is expected that continuum breakdown will occur in areas of high gradients, in the shock wave and boundary layer, and in areas of rarefied gas flow in the wake. It is also expected that the amount of continuum break down will increase with increasing Mach and Knudsen number. Also, a comparison of the present results to the cylinder case will be made in order to investigate any changes in continuum breakdown due to axisymmetry.

The results that are presented in this paper for the surface aerothermodynamic properties are given as non-dimensionalized coefficients which are defined by Eqs. $(10-12)$.

$$
\begin{aligned}
C_{p} & =\frac{p-p_{\infty}}{\frac{1}{2} \rho_{\infty} U_{\infty}^{2}} \\
C_{\tau} & =\frac{\tau}{\frac{1}{2} \rho_{\infty} U_{\infty}^{2}} \\
C_{q} & =\frac{q}{\frac{1}{2} \rho_{\infty} U_{\infty}^{3}}
\end{aligned}
$$

where $\mathrm{p}$ is the pressure, $\tau$ is the shear stress, $\mathrm{q}$ is the heat flux, $\rho_{\infty}$ is the free stream density and $U_{\infty}$ is the free stream velocity. The surface aerothermodynamic properties are plotted against the surface angle $\phi$, which is measured from the stagnation point.

\section{IV.A. Rotational Nonequilibrium}

To test the validity of the rotational energy model the temperature profiles from CFD and DSMC along the stagnation stream line are compared in Fig. 2. Also included in these figures is the maximum gradient length local Knudsen number based on the DSMC simulation. This will give an idea of the amount of breakdown in the flow, but also gives a good approximate location of the shock. For the Mach 10 Knudsen number 0.002 case the shock is very clearly defined by $K n_{G L L}$, and it can be seen that the translational temperature, shown as solid lines, predicted by DSMC starts increasing further upstream due to the fact that the shock predicted by DSMC is thicker, as expected. However, the translational temperature from CFD quickly matches the temperature from DSMC although it does not capture the peak. It can be seen that there is rotational nonequilibrium behind the shock for both DSMC and CFD, but both rotational temperatures, shown as dashed lines, nearly overlap each other along the stagnation streamline. For the Mach 10 Knudsen number 0.01 case, the shock is not as clearly defined and $K n_{G L L}$ is greater than the 0.05 limit from the shock all the way to the wall, indicating continuum breakdown has occurred. In this case the difference in the shock thickness is much more noticeable, but CFD quickly catches up and then slightly over predicts the translational temperature. There is rotational nonequilibrium behind the shock for both CFD and DSMC, and again the difference in shock thickness leads to a difference in the rotational temperatures. However, the rotational temperature predicted by CFD catches and overshoots the DSMC rotational temperature post shock and eventually all temperatures equilibrate. This discrepancy can be explained by the fact that the flow is in nonequilibrium following the shock, which is verified by looking at velocity distribution functions at various points along the stagnation streamline. At the point where the CFD temperatures overshoot the DSMC temperatures there is still a bimodal distribution indicating that the flow is in nonequilibrium.

Figures 2(c) and (d) give the temperature profiles for Mach 25 and 45 at a global Knudsen number of 0.01. At these higher Mach numbers, the simulations now include vibrational as well as rotational nonequilibrium. The Mach 25 case is similar to the Mach 10 case in that there is breakdown in the flow from the front of the shock all the way to the wall of the sphere. The difference in the shock thickness is again visible in the temperature profiles for DSMC and CFD, but CFD quickly catches up and again slightly over predicts the translational temperature. The vibrational temperature, shown as dotted lines, and the rotational temperature as predicted by CFD both overshoot their DSMC counterparts. However, all temperatures for both DSMC and CFD fall back into equilibrium at approximately the same point. For the Mach 45 case, the slight over prediction by CFD for all the temperatures still exists, but again all three temperatures reach equilibrium at the same point. Since the Mach number is so high in this case, the point that equilibrium is reached is pushed close to the wall of the sphere. The overshoots in the temperature are again caused by the fact that the flow is in nonequilibrium. 


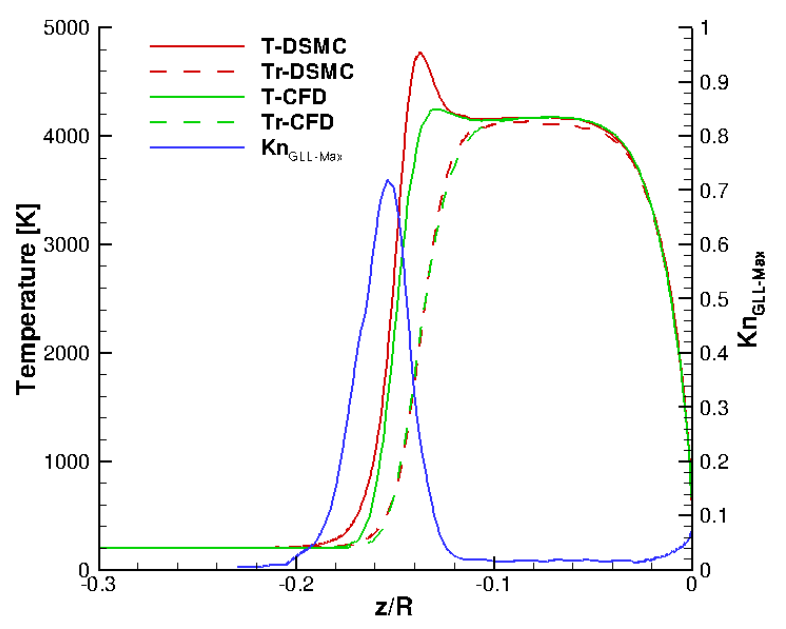

(a) Mach $10, K n_{\infty}=0.002$

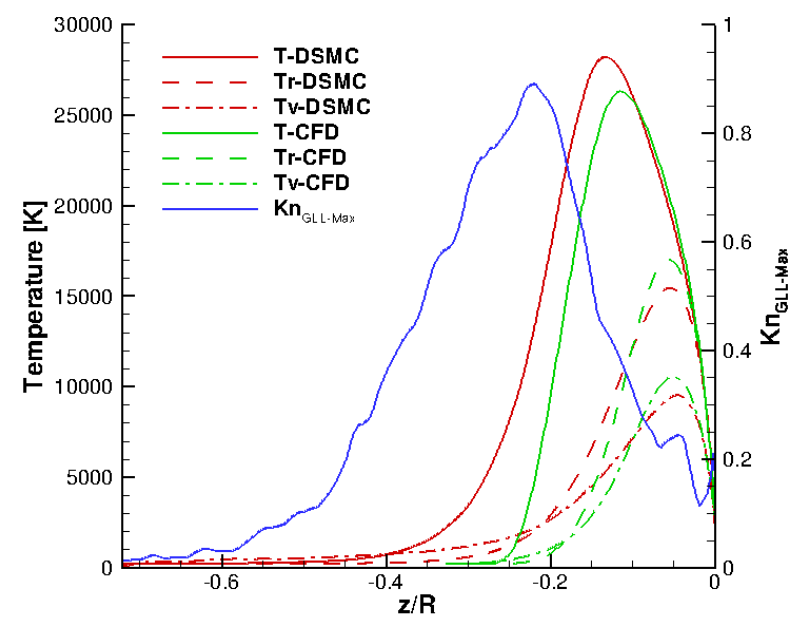

(c) Mach $25, K n_{\infty}=0.01$

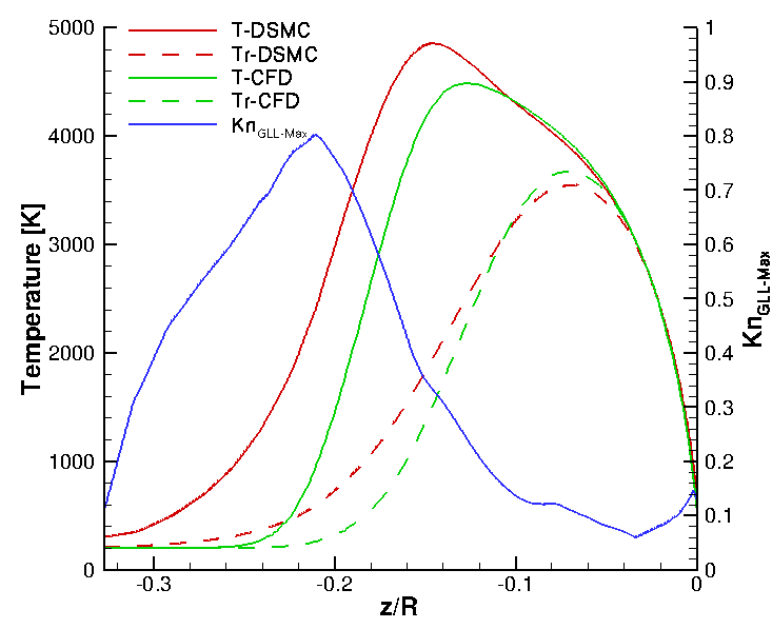

(b) Mach $10, K n_{\infty}=0.01$

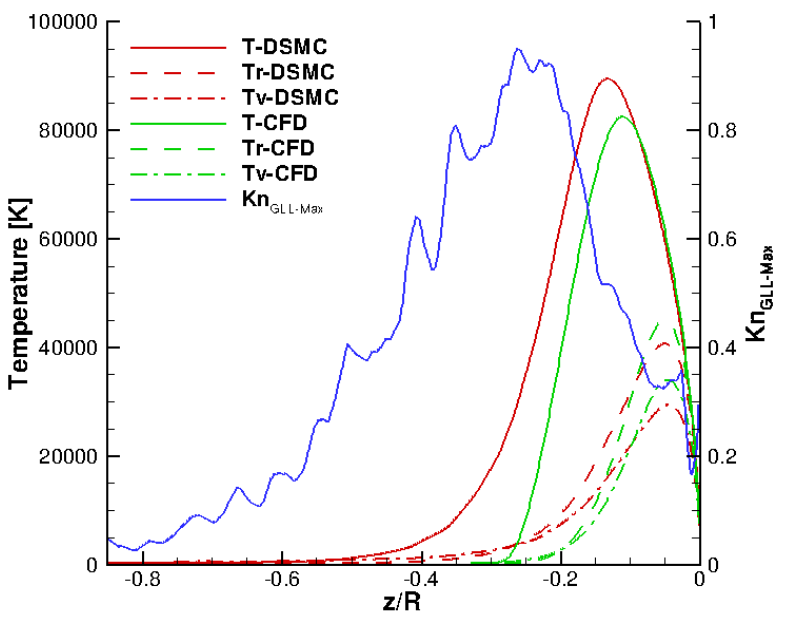

(d) Mach $45, K n_{\infty}=0.01$

Figure 2. Temperature profiles along the stagnation line. The maximum $K n_{G L L}$ is plotted on the right axis. Flow is from left to right; distance is normalized by the radius of the sphere. 


\section{IV.B. Effect of Global Knudsen Number}

To begin with, the results for the Mach 10 simulations at Knudsen numbers of 0.002 to 0.25 are discussed in detail. The goal is to be able to discern the effects of changing the Knudsen number on continuum breakdown. The integrated drag and peak heat flux are given in Tables 2 and 3, respectively. The tables give the values predicted by DSMC and CFD along with a percent difference between the two. Since DSMC is a particle method that works in both the continuum regime and the rarefied regime it is assumed that the DSMC results are more accurate, so the percent difference is calculated using the DSMC result as the correct result. At the lowest Knudsen number the integrated drag predictions are nearly identical and the peak heat flux values are close. However, as the Knudsen number grows, the results diverge until the differences between DSMC and CFD are $28 \%$ and $58 \%$ for integrated drag and peak heat flux at the highest Knudsen number, respectively.

Table 2. Integrated Drag [N] from DSMC and CFD for Mach 10 Flow

\begin{tabular}{|c|c|c|c|}
\hline$K n_{\infty}$ & DSMC & CFD & Percent Difference \\
\hline \hline 0.002 & 147.66 & 147.42 & $-0.166 \%$ \\
0.01 & 30.78 & 31.62 & $2.69 \%$ \\
0.05 & 7.26 & 7.7 & $5.98 \%$ \\
0.25 & 3.44 & 2.46 & $28.26 \%$ \\
\hline
\end{tabular}

Table 3. Peak Heating $\left[\frac{W}{m^{2}}\right]$ from DSMC and CFD for Mach 10 Flow

\begin{tabular}{|c|c|c|c|}
\hline$K n_{\infty}$ & DSMC & CFD & Percent Difference \\
\hline \hline 0.002 & $1.09 \times 10^{5}$ & $1.15 \times 10^{5}$ & $5.18 \%$ \\
0.01 & $4.65 \times 10^{4}$ & $5.46 \times 10^{4}$ & $17.38 \%$ \\
0.05 & $2.11 \times 10^{4}$ & $2.54 \times 10^{4}$ & $20.68 \%$ \\
0.25 & $6.43 \times 10^{3}$ & $1.02 \times 10^{4}$ & $58.23 \%$ \\
\hline
\end{tabular}

From the integrated drag and the total heat flux there is a correlation between increasing Knudsen number and increasing difference between DSMC and CFD results. So the question becomes, how does increasing global Knudsen number correspond to continuum breakdown. This question can be answered by looking at how $K n_{G L L}$ varies with global Knudsen number, presented in Fig. 3. This figure shows contours of $K n_{G L L}=0.05$ for global Knudsen numbers from 0.002 to 0.25 . It is seen that as the global Knudsen number increases there is a larger amount of continuum breakdown occurring in the flow. It should be noted that at a global Knudsen number of 0.25 , nearly the entire domain has $K n_{G L L}$ above 0.05 , only at the very back of the domain can the contour be seen. Although the free stream conditions are not in breakdown, that cannot be seen because the shock for the Knudsen 0.25 case is beyond the range of this diagram. The $K n_{G L L}$ contour for a global Knudsen number of 0.002 can be seen as a very thin line at the shock, but there is still a significant amount of breakdown in the wake. In the subsections to follow, the surface properties and $K n_{G L L}$ are discussed in further detail.

\section{IV.B.1. $K n_{\infty}=0.002$}

At a global Knudsen number of 0.002 the flow is expected to be well within the continuum regime, therefore CFD should have no problem properly simulating this flow. Even though the free stream conditions are in the continuum regime, there is still continuum breakdown in the flow, as shown in Fig. 4(a). This figure shows that there is continuum breakdown in the shock and in the wake of the sphere. At this global Knudsen number, CFD and DSMC give approximately the same amount of continuum breakdown in the flow, even the shocks predicted by DSMC and CFD have the same thickness.

The surface coefficient of pressure is given in Fig. 4(b). It can be seen that the pressure predicted by DSMC and CFD agree very well. There is only a very small difference at the stagnation point and in the wake of the flow, caused by breakdown in the wake of the sphere. A theoretical calculation of the coefficient of stagnation pressure, found in the inviscid limit to be 1.825, compares well with the numerical prediction of 


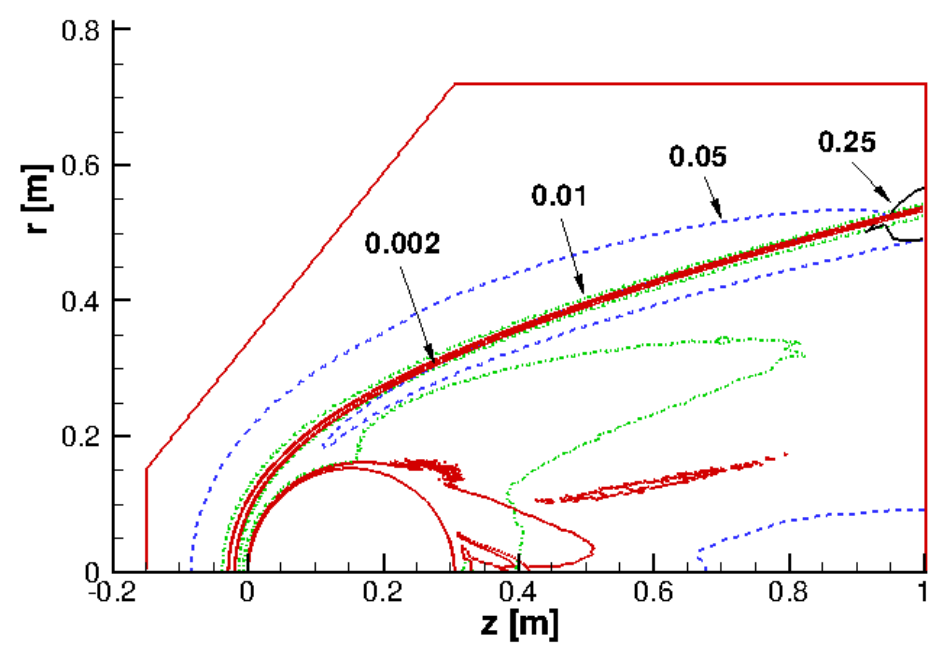

Figure 3. $K n_{G L L}=0.05$ for varying global Knudsen number at Mach 10

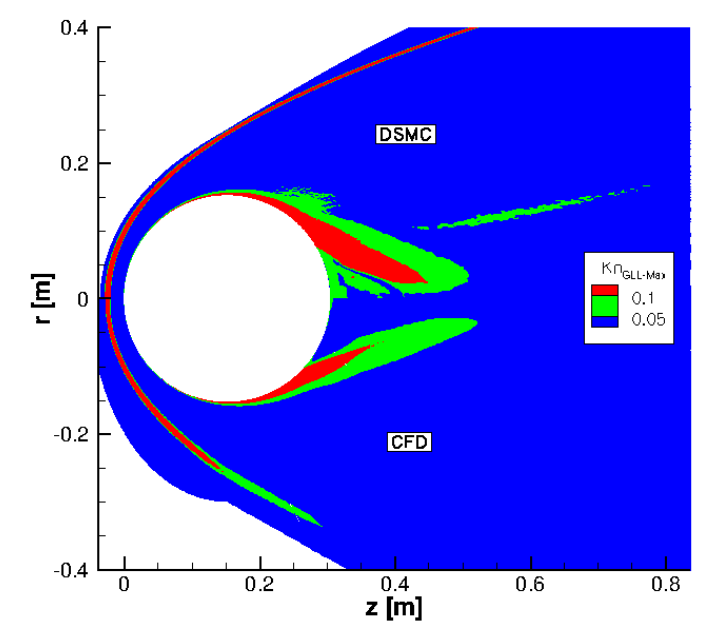

(a) $K n_{G L L}$

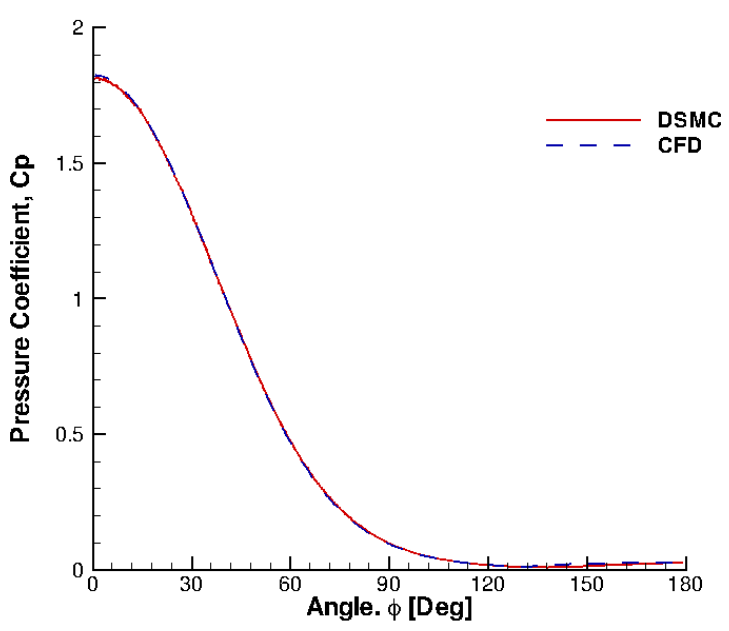

(b) Coefficient of Pressure

Figure 4. $K n_{\infty}=0.002, K n_{G L L}$ and surface pressure on a sphere in a Mach 10 flow of nitrogen 
1.823. The shear stress along the surface, as given in Fig. 5(b), compares very well over most of the surface, although the DSMC curve is very noisy but this is to be expected due to the relatively high density. The CFD and DSMC predictions for shear stress coefficient start to diverge over the backside of the sphere, which is to be expected since there is a large area of breakdown in the wake. It can be seen that the divergence in surface shear stress coefficient begins at approximately the same point where the breakdown becomes significant at around 90 degrees. Even with the difference in the shear stress in the wake of the body, the integrated drag compares very well with less then 0.2 percent difference between CFD and DSMC. The heat flux coefficient over the surface of the sphere is given in Fig. 5(a). The CFD prediction of surface heat flux coefficient is higher then DSMC over most of the surface, but both go to near zero over the back side of the sphere. The largest difference between CFD and DSMC predictions of heat transfer rate occurs near the stagnation point where CFD is almost 5 percent higher than DSMC. Figure 2(a) shows that $K n_{G L L}=0.05$ at the stagnation point, which means breakdown has occurred and may explain the 5 percent difference in the peak heat transfer rate.

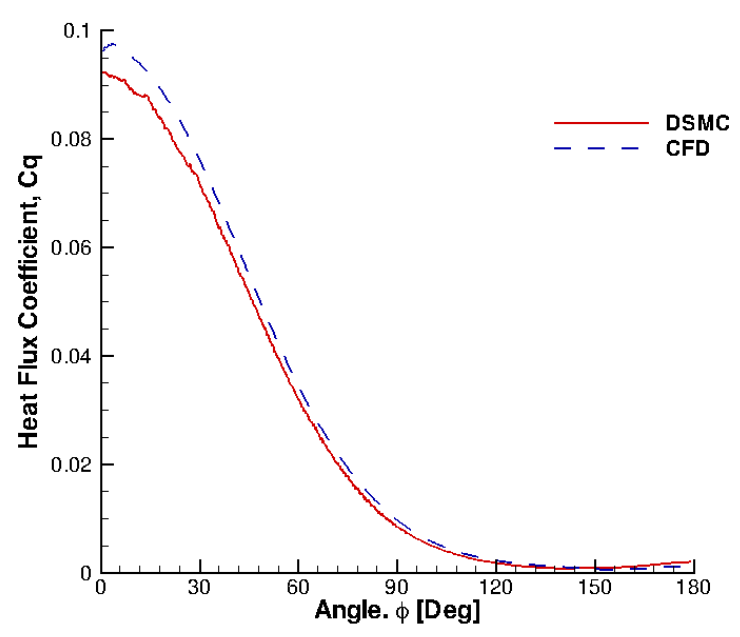

(a) Coefficient of Heat Flux

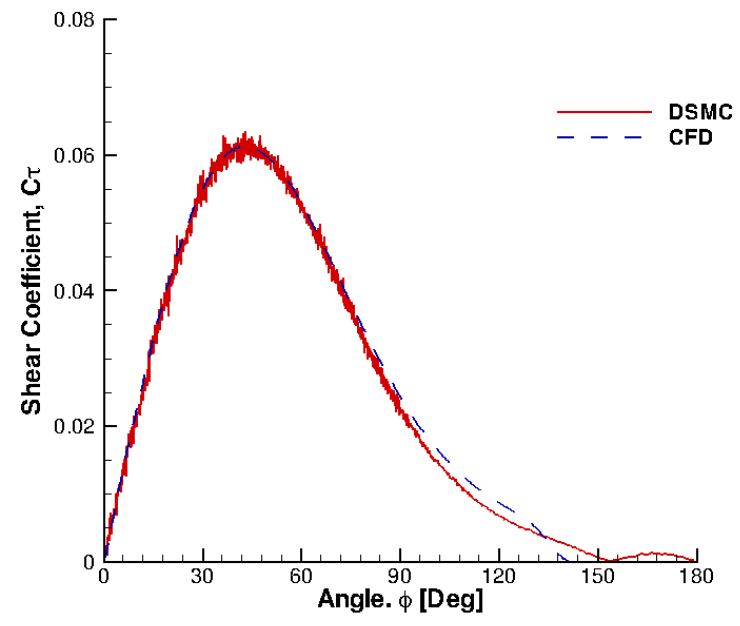

(b) Coefficient of Shear Stress

Figure 5. $K n_{\infty}=0.002$, surface heat flux and shear stress on a sphere in a Mach 10 flow of nitrogen

\section{IV.B.2. $K n_{\infty}=0.01$}

The traditional, but often argued about, limit for accurate CFD simulations is a Knudsen number of 0.01. At a Knudsen number of 0.01 the flow is on the edge of the continuum regime, but the flow is not yet rarefied. From Fig. 6(a) it can be seen from the contours of $K n_{G L L}$ that there is breakdown occurring in the shock, near the wall and in the wake of the sphere. It should be noted that at this global Knudsen number, DSMC predicts a larger amount of breakdown in the wake than CFD. Also, at this condition, the shock is noticeably thicker in the DSMC case while the location of the shock is approximately the same for DSMC and CFD.

Even at this higher global Knudsen number, the pressures from DSMC and CFD still agree very well. However, there is a small difference between CFD and DSMC near the stagnation point where CFD is higher than DSMC. For this case the flow is in continuum breakdown from the shock all the way to the wall on the stagnation streamline, which may explain the discrepancies in pressure. The surface heat flux coefficient predicted by CFD is always larger than DSMC as shown in Fig. 7(a). At this Knudsen number, the heat flux coefficient predicted by CFD gives a positive value over the aft surface of the sphere while DSMC predicts a near zero heat flux coefficient. However, the largest difference between CFD and DSMC heat transfer rate occurs near the stagnation point where CFD is 17 percent higher than DSMC. The shear stress coefficient, given in Fig. 7(b), compares well between the two techniques near the stagnation region but then begins to diverge over the latter part of the surface. From the plot of the shear stress coefficient it can be seen that while DSMC predicts a lower magnitude at the peak, the peak for both DSMC and CFD occur at approximately the same location. Given that a large amount of continuum breakdown occurs in the wake of the sphere it is not surprising that the shear stress coefficients from CFD and DSMC disagree over the latter 


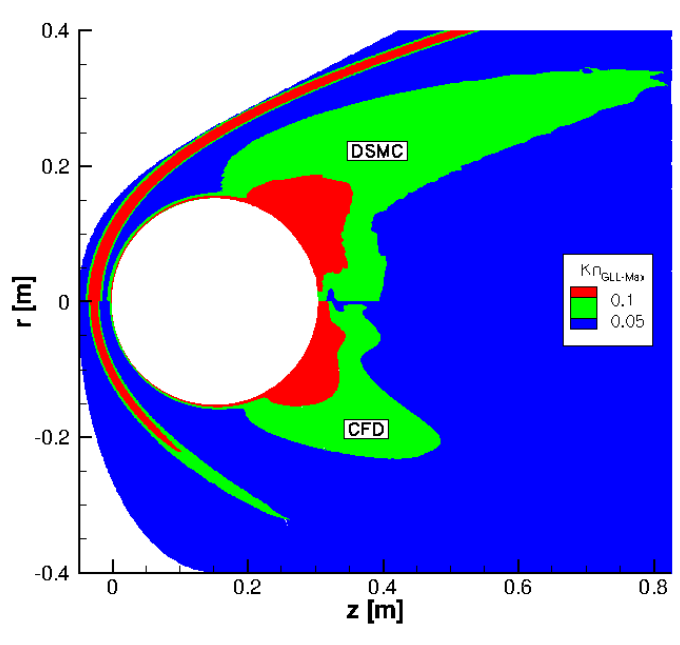

(a) $K n_{G L L}$

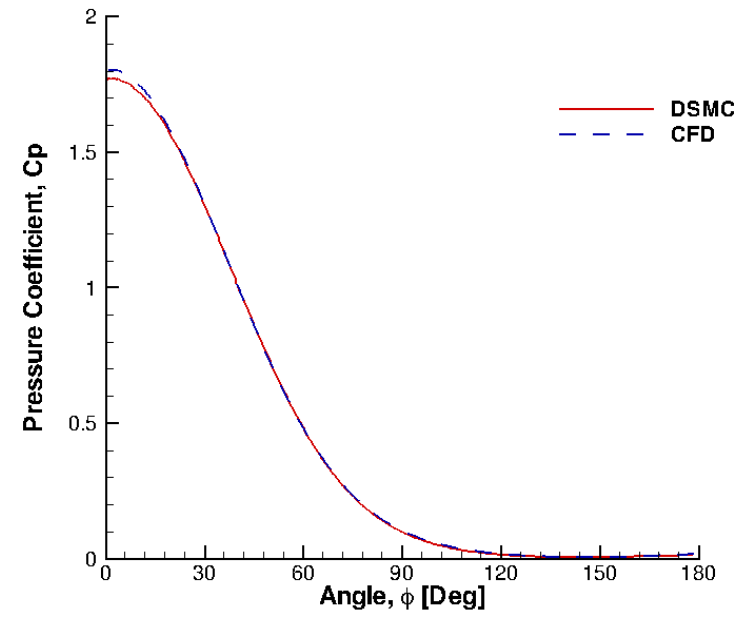

(b) Coefficient of Pressure

Figure 6. $K n_{\infty}=0.01, K n_{G L L}$ and surface pressure on a sphere in a Mach 10 flow of nitrogen

half of the sphere. For the integrated drag, CFD is 3 percent higher than DSMC at this condition. For this case it is easy to notice that the shear and heat flux on the surface are higher in CFD than DSMC, that is due to a no velocity slip boundary stipulation and a no temperature jump boundary condition in CFD.

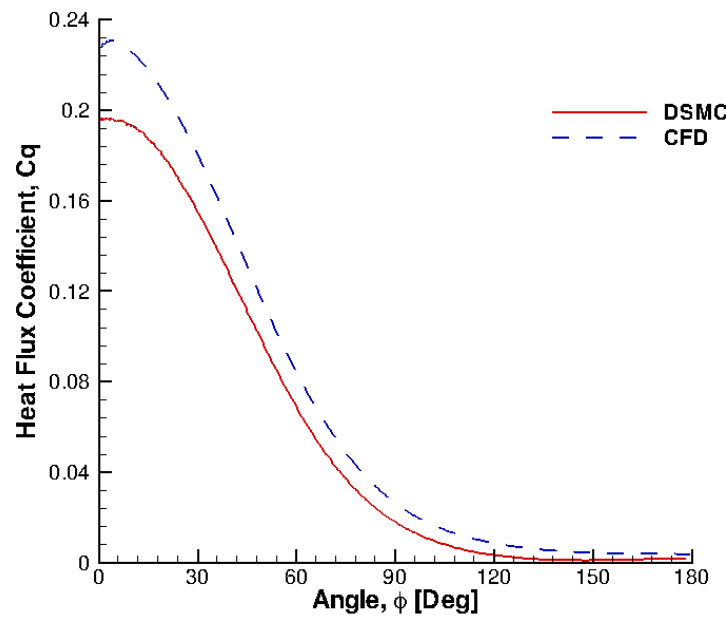

(a) Coefficient of Heat Flux

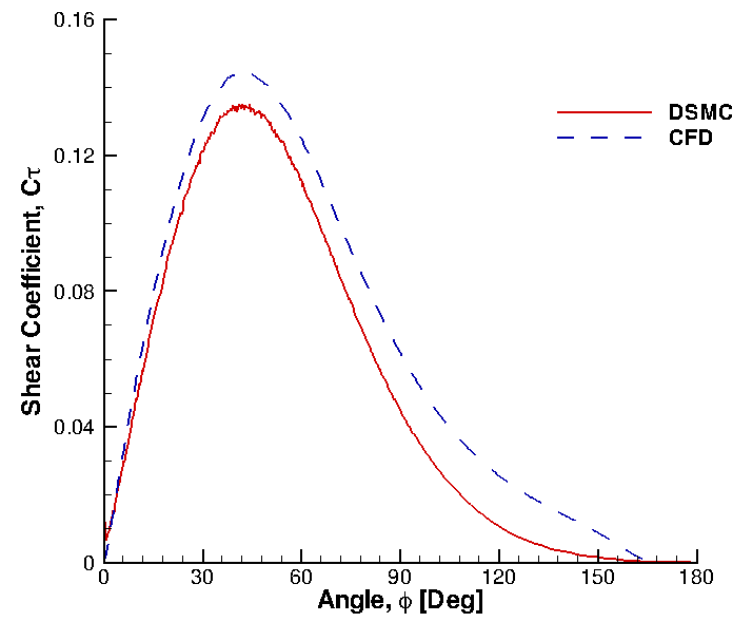

(b) Coefficient of Shear Stress

Figure 7. $K n_{\infty}=0.01$, Surface heat flux and shear stress on a sphere in a Mach 10 flow of nitrogen

\section{IV.B.3. $K n_{\infty}=0.05$}

At a global Knudsen number of 0.05 , the flow is outside the supposed limit for physically accurate CFD simulations, moving out of the continuum regime and approaching a rarefied gas. The flow does show that there is a large amount of breakdown in the shock, boundary layer and wake, as seen in Fig. 8(a). From this figure it is seen that $K n_{G L L}$ exceeds the critical value of 0.05 for a large part of the domain for both CFD and DSMC. This means that continuum breakdown has occurred and CFD should have difficulty simulating the flow accurately. It is interesting to note that the shock has become thicker in DSMC than CFD, but the shock standoff distance is approximately the same for both methods. While the amount of breakdown in the 
shock is approximately the same it can be seen that the breakdown predicted by CFD is larger in the wake.

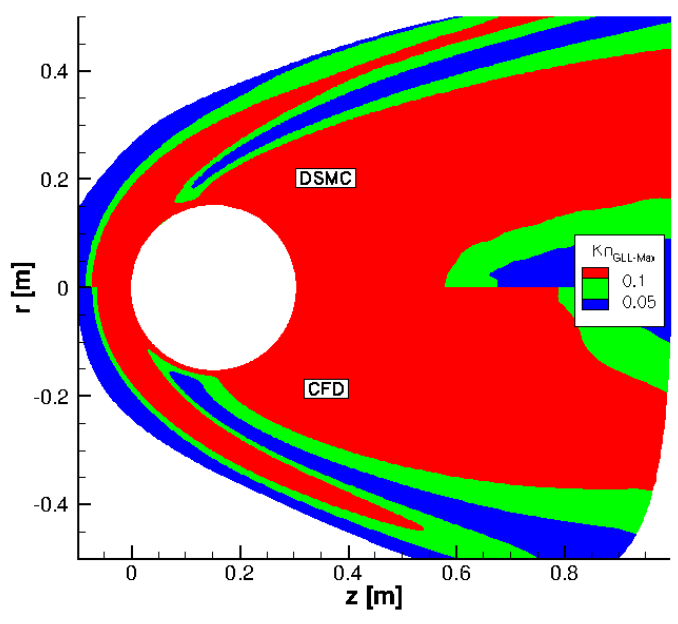

(a) $K n_{G L L}$

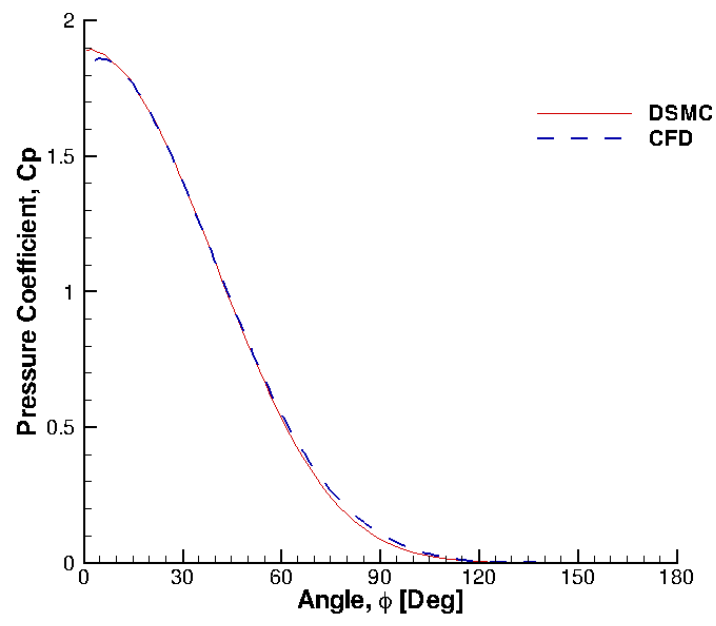

(b) Coefficient of Pressure

Figure 8. $K n_{\infty}=0.05, K n_{G L L}$ and surface pressure on a sphere in a Mach 10 flow of nitrogen

The surface pressure on the sphere simulated by CFD and DSMC is still in very good agreement as shown in Fig. 8(b). Even as the Knudsen number grows, the pressure predicted by both methods shows good agreement. Notice that the DSMC and CFD predictions of pressure diverge slightly near the stagnation point and in the wake of the sphere. Not surprisingly this coincides with where the breakdown is strongest in the flow. One oddity is that the CFD prediction of pressure is less than DSMC at the stagnation point, which is opposite from what is seen in other cases. The surface heat flux, as seen in Fig. 9(a), displays a large separation between CFD and DSMC. This separation starts at the stagnation point and goes all the way to the backside of the sphere. Notice that the heat transfer coefficient predicted by CFD in the wake is positive and greater than that for the global Knudsen number 0.01 case. The difference between CFD and DSMC predictions of heat transfer rate is highest at the stagnation point where the CFD is 20 percent higher than DSMC. The shear stress, given in Fig. 9(b), for both DSMC and CFD agree very well over the first 25 degrees, but then the two diverge over the rest of the surface due to the growing amount of breakdown. The CFD prediction for the surface shear stress gives the peak value at a slightly later point on the surface and gives a value higher than DSMC does. The CFD results for integrated drag are 6 percent higher than DSMC.

\section{IV.B.4. $K n_{\infty}=0.25$}

The last, and highest global Knudsen number, that is discussed in this work is 0.25 . This gives a flow that is well outside of the continuum regime and is now a rarefied gas. At this Knudsen number, the flow is nearly entirely in continuum breakdown, as seen in Fig. 10(a). It is seen that $K n_{G L L}$ exceeds the critical value of 0.05 for almost the entire domain for both CFD and DSMC. There is only a small portion that is not in continuum breakdown behind the sphere for DSMC. At this global Knudsen number, the shock is very far out in front of the sphere, $0.4 \mathrm{~m}$ from the stagnation point, while the other cases are all less than $0.1 \mathrm{~m}$ from the stagnation point.

At a global Knudsen number of 0.25 , the flow is rarefied and as a result the entire flow is considered to be in continuum breakdown, which has a significant effect on the surface properties of the sphere. Figure 10(b) gives the coefficient of pressure along the surface of the sphere. From this figure, CFD over predicts the pressure over most of the sphere; after about 120 degrees they both go to near zero. It is interesting to note that at this global Knudsen number, the CFD predicted coefficient of pressure goes above 2 at the stagnation point. A theoretical calculation of the coefficient of stagnation pressure, found in the free molecular limit to be 2.13, shows that a value near two is still within reason at this high a global Knudsen number. The surface heat flux coefficient, given in Fig. 11(a), shows that there is a large difference between DSMC and CFD over the whole surface of the sphere. CFD predicts a positive heat flux coefficient on the aft surface 


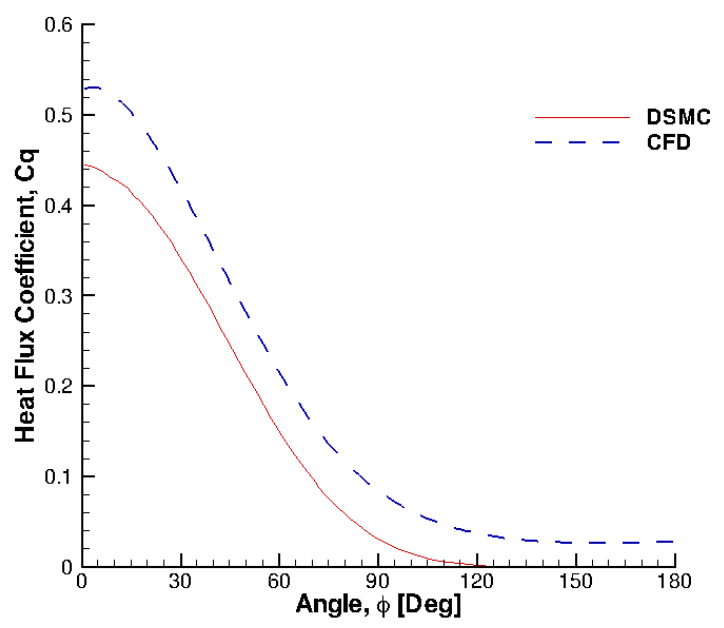

(a) Coefficient of Heat Flux

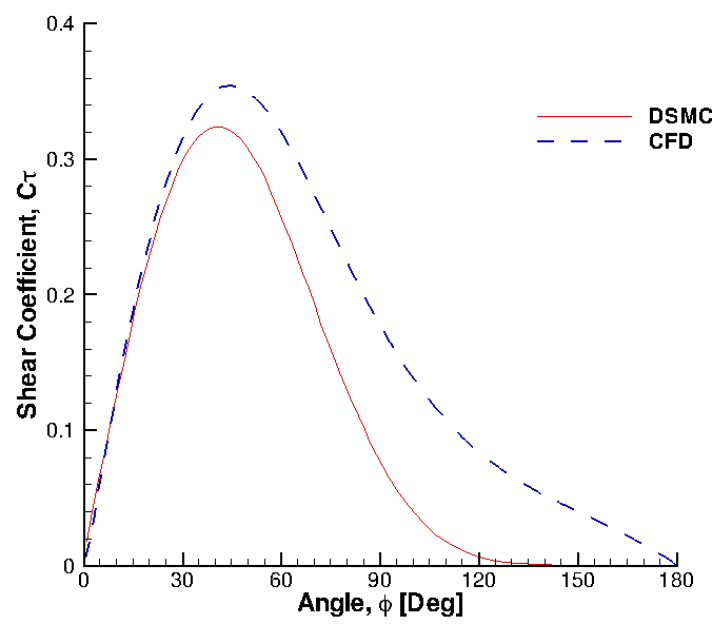

(b) Coefficient of Shear Stress

Figure 9. $K n_{\infty}=0.05$, Surface heat flux and shear stress on a sphere in a Mach 10 flow of nitrogen

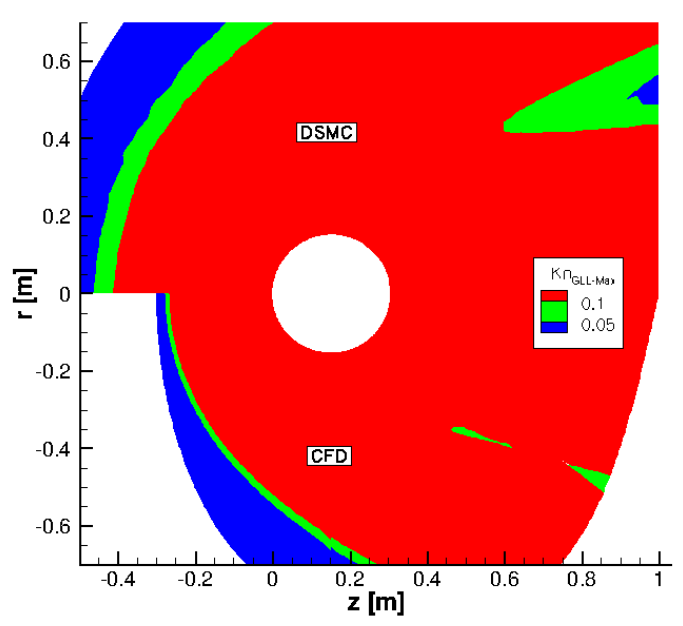

(a) $K n_{G L L}$

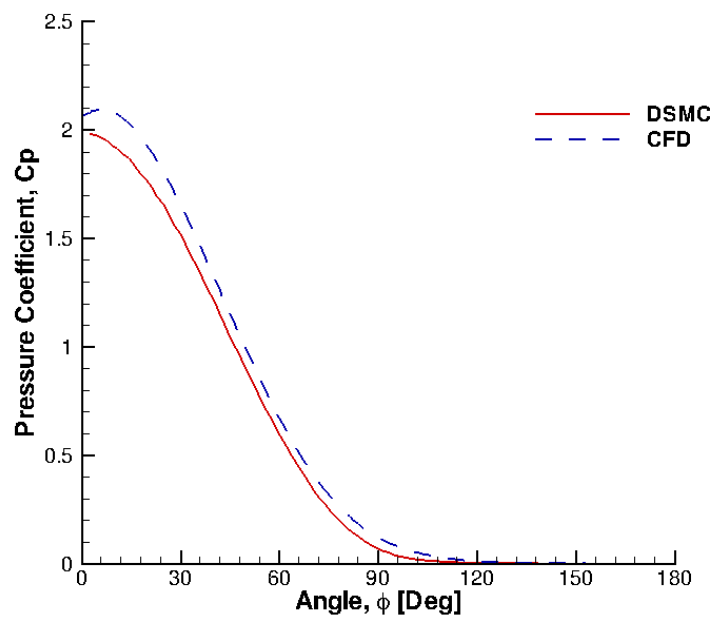

(b) Coefficient of Pressure

Figure 10. $K n_{\infty}=0.25, K n_{G L L}$ and surface pressure on a sphere in a Mach 10 flow of nitrogen 
of the sphere while DSMC predicts near zero heat flux coefficient. The difference between CFD and DSMC predicted values of heat flux coefficient over the entire sphere, but most noticeably on the aft of the sphere, has increased with increasing Knudsen number. The largest difference between CFD and DSMC predictions of heat transfer rate occurs near the stagnation point where CFD is almost 60 percent higher than DSMC. Figure 11(b) shows the shear stress coefficient along the surface of the sphere. The shear stresses agree well near the stagnation point, but then DSMC and CFD start to diverge. The shear stress predicted by DSMC gives a peak at a smaller angle and at a much smaller magnitude than CFD. These differences persist all the way to the rear stagnation point of the sphere. For this case, the integrated drag is 28 percent higher in CFD than DSMC.

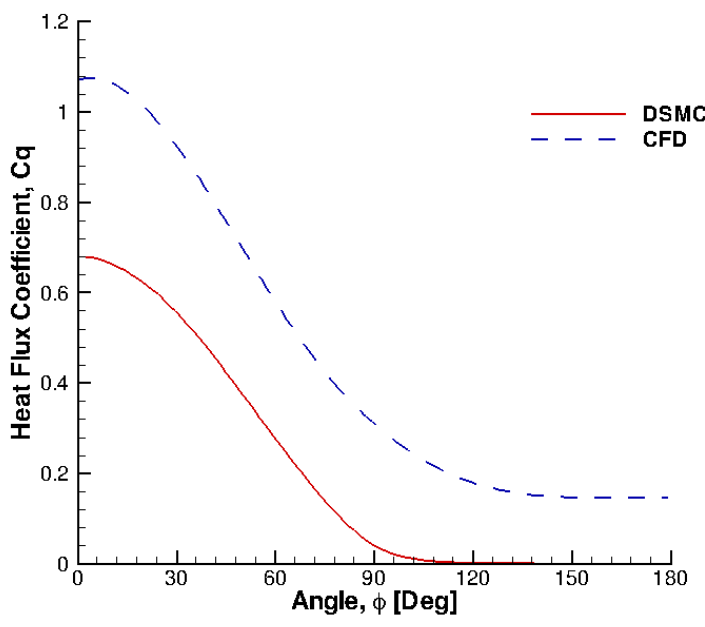

(a) Coefficient of Heat Flux

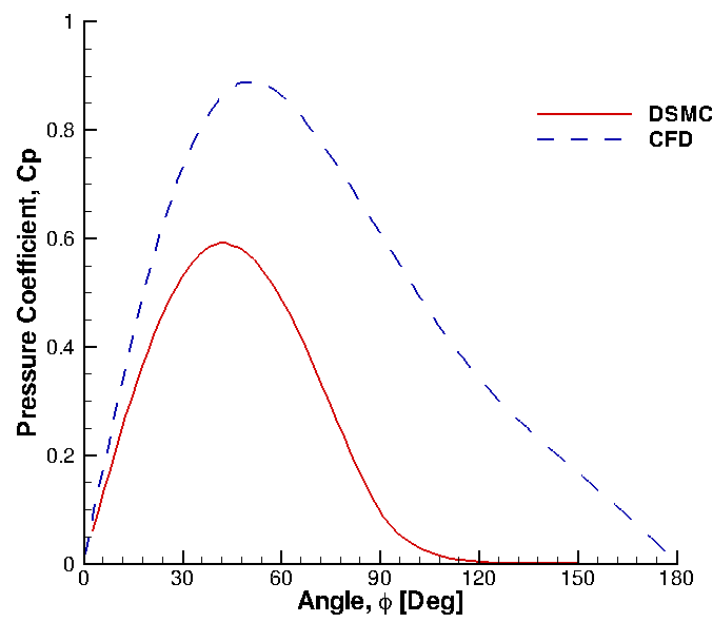

(b) Coefficient of Shear Stress

Figure 11. $K n_{\infty}=0.25$, Surface heat flux and shear stress on a sphere in a Mach 10 flow of nitrogen

\section{IV.C. Effect of Mach Number}

The next set of results discussed is from simulations at a global Knudsen number of 0.01 for Mach numbers of 10, 25, and 45. This study aims to discern the effects of changing the Mach number on continuum breakdown, while freezing the global Knudsen number. Since the Mach 10 simulation was discussed as part of the results in the last section it will not be discussed in this section. Therefore, this section begins by discussing the results of Mach 25 at a global Knudsen number of 0.01. The integrated drag and peak heat flux are given in Tables 4 and 5, respectively. The tables give the values predicted by DSMC and CFD along with a percent difference between DSMC and CFD. Once again, it is assumed that the DSMC results are more accurate, so the percent difference is calculated using the DSMC result. Even at Mach 10 there is a sizable difference between CFD and DSMC for the integrated drag and total heat flux. It is interesting to note that the differences do not increase with increasing Mach number.

Table 4. Integrated Drag [N] for DSMC and CFD for a Knudsen Number of 0.01

\begin{tabular}{|c|c|c|c|}
\hline Mach Number & DSMC & CFD & Percent Difference \\
\hline \hline 10 & 30.78 & 31.62 & $2.69 \%$ \\
25 & 198.1 & 203.18 & $2.52 \%$ \\
45 & 650.22 & 670.32 & $3.09 \%$ \\
\hline
\end{tabular}

From the integrated drag and the total heat flux there does not appear to be a correlation between Mach number and the differences between DSMC and CFD results. However, this does not mean that Mach number has not affected continuum breakdown. This is seen by looking at how $K n_{G L L}$ varies with Mach number, as presented in Fig. 12. This figure shows contours of $K n_{G L L}=0.05$ for the three Mach numbers. 
Table 5. Peak Heating $\left[\frac{W}{m^{2}}\right]$ for DSMC and CFD for a Knudsen Number of 0.01

\begin{tabular}{|c|c|c|c|}
\hline Mach Number & DSMC & CFD & Percent Difference \\
\hline \hline 10 & $4.65 \times 10^{4}$ & $5.46 \times 10^{4}$ & $17.38 \%$ \\
25 & $9.81 \times 10^{5}$ & $1.14 \times 10^{6}$ & $16.02 \%$ \\
45 & $6.84 \times 10^{6}$ & $8.01 \times 10^{6}$ & $17.13 \%$ \\
\hline
\end{tabular}

It is seen that as the Mach number increases, the area of flow in continuum breakdown increases. In the subsections to follow, the surface properties and $K n_{G L L}$ are discussed in further detail.

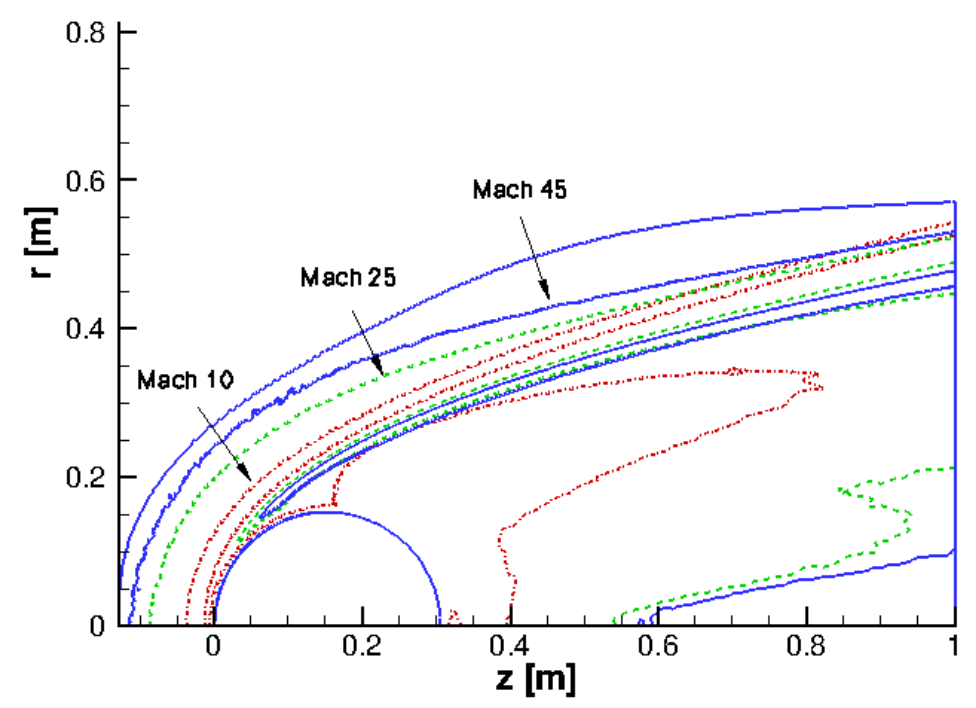

Figure 12. $K n_{G L L}$ constant contours of 0.05 for varying Mach number at a global Knudsen number of 0.01

\section{IV.C.1. Mach 25}

For this test case, the Knudsen number is kept at 0.01 while the Mach number is increased to 25. At this Mach number, there is rotational and vibrational nonequilibrium. From Fig. 13(a) it is seen that there is breakdown occurring in the shock, near the wall and in the wake of the sphere. It should be noted that at this Mach number DSMC predicts a larger amount of breakdown in the wake, and there is breakdown in the flow from the shock all the way to the wall around the stagnation streamline. Also, the amount of continuum breakdown predicted by DSMC is greater than the amount of breakdown predicted by CFD.

Even at a higher Mach number, the pressures from DSMC and CFD still agree very well, as seen in Fig. 13(b). However, there is again a small difference near the stagnation point where the simulated coefficient of pressure in CFD is higher than DSMC. The surface heat flux coefficient, given in Fig. 14(a), predicted by CFD is always larger than DSMC. At Mach 25, the heat flux coefficient simulated by CFD gives a positive value over the aft surface of the sphere, much like the Mach 10 case. However, the largest difference between CFD and DSMC heat transfer rate occurs near the stagnation point where CFD is 16 percent higher than DSMC. The shear stress coefficient, given in Fig. 14(b), compares well between the two techniques near the stagnation region but then begins to diverge over the latter part of the sphere. From the plot of the shear stress coefficient it is seen that while DSMC has a lower magnitude at the peak, the peak for both DSMC and CFD occur at approximately the same location. For the integrated drag, CFD is higher by 3 percent than DSMC. The results for this case are very similar to the Mach 10 case, even though the Mach number more than doubled. 


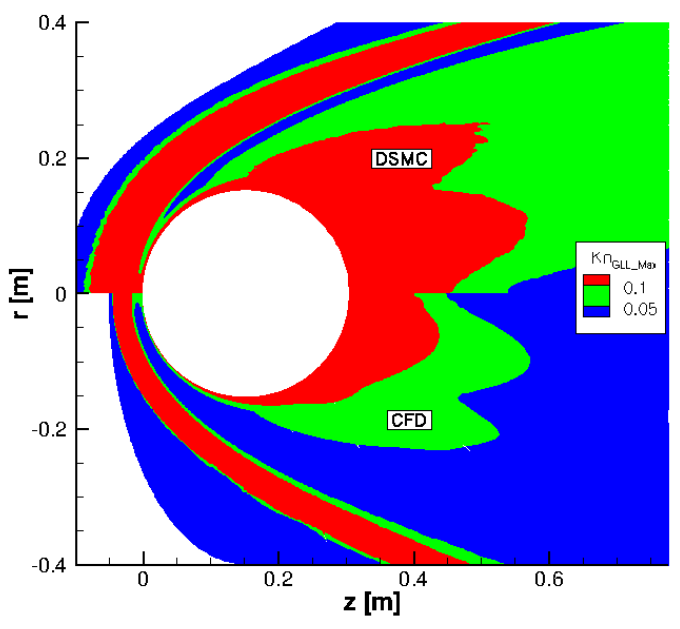

(a) $K n_{G L L}$

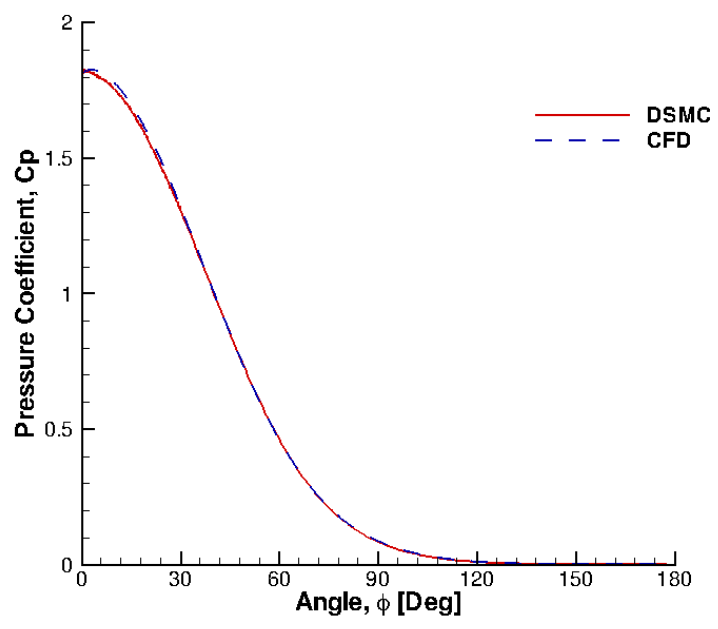

(b) Coefficient of Pressure

Figure 13. $K n_{\infty}=0.01, K n_{G L L}$ and surface pressure on a sphere in a Mach 25 flow of nitrogen

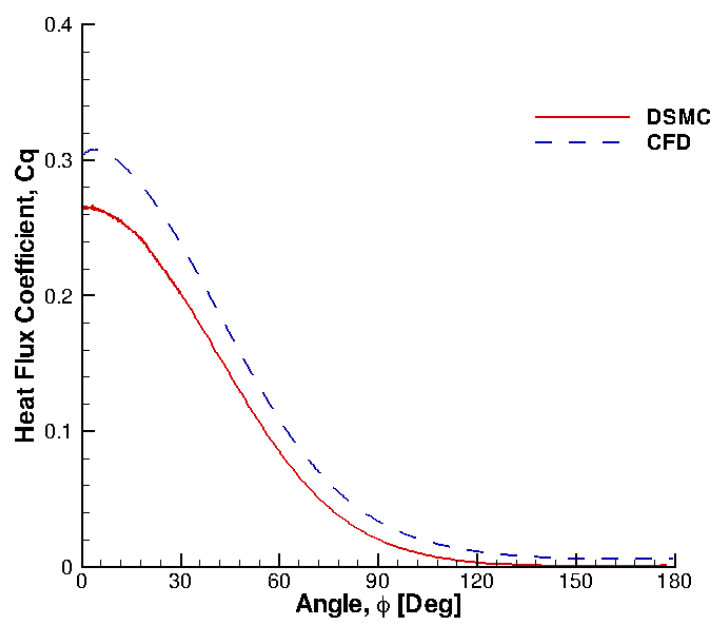

(a) Coefficient of Heat Flux

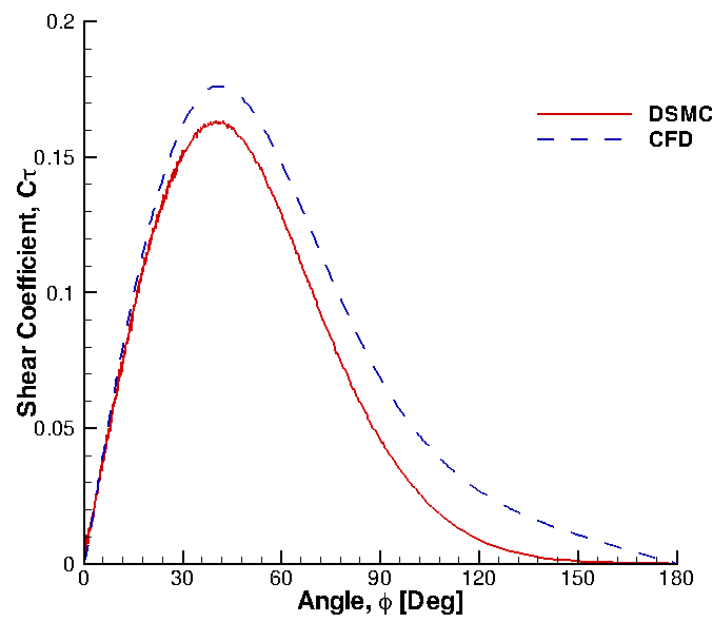

(b) Coefficient of Shear Stress

Figure 14. $K n_{\infty}=0.01$, Surface heat flux and shear stress on a sphere in a Mach 25 flow of nitrogen 


\section{IV.C.2. Mach 45}

At this Mach number there is breakdown in the shock and the wake of the flow as seen in Fig. 15(a), and again the flow from the shock all the way to the wall along the stagnation stream line is in continuum breakdown. From the figure it is seen that the amount of breakdown predicted by DSMC is larger in the shock region and in the wake than predicted by CFD.

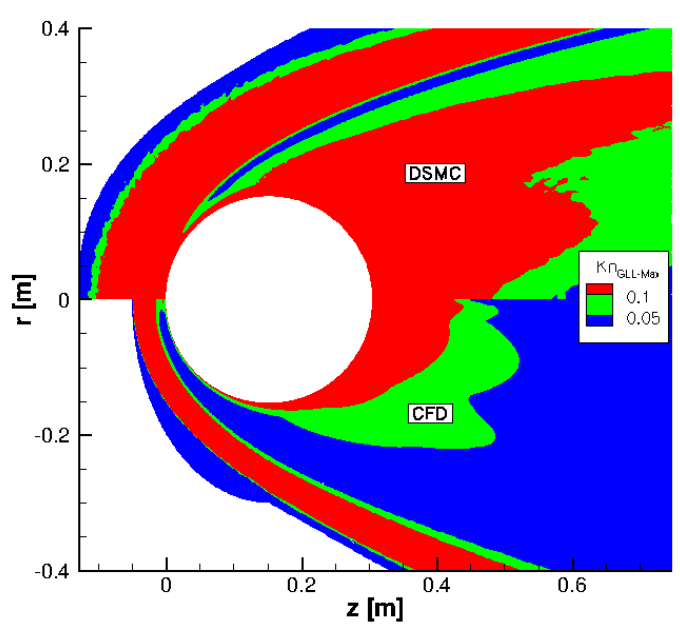

(a) $K n_{G L L}$

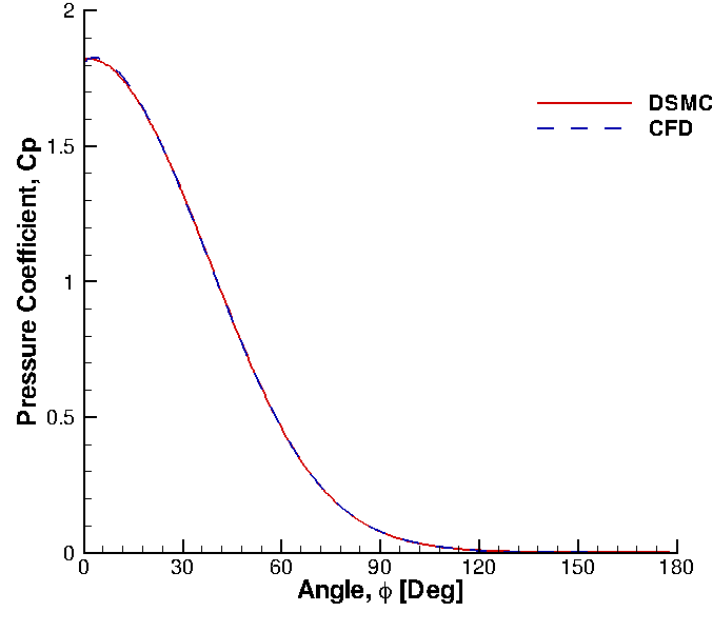

(b) Coefficient of Pressure

Figure 15. $K n_{\infty}=0.01, K n_{G L L}$ and surface pressure on a sphere in a Mach 45 flow of nitrogen

The surface pressure coefficient, given in Fig. 15(b), for DSMC and CFD match very well over the surface of the sphere. The surface heat flux coefficient displays a gap between CFD and DSMC over the entire surface of the sphere, as shown in Fig. 16(a). The largest difference between CFD and DSMC for the heat flux occurs near the stagnation point where there is a 17 percent difference. The surface shear stress, as given in Fig. 16(b), for CFD and DSMC agree well over the beginning of the sphere. The CFD shear stress has a higher magnitude at the peak, but both methods predict the peak at nearly the same location. After the peak, the CFD and DSMC simulations of the shear stress coefficient diverge.

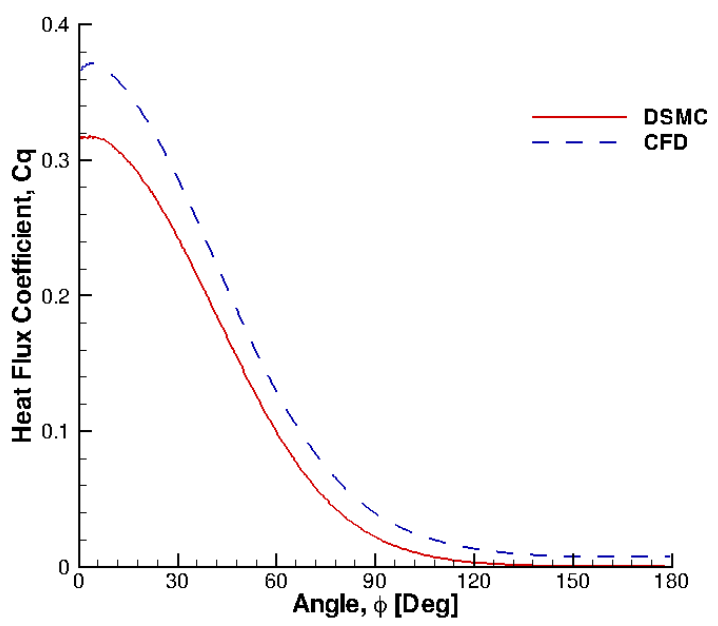

(a) Coefficient of Heat Flux

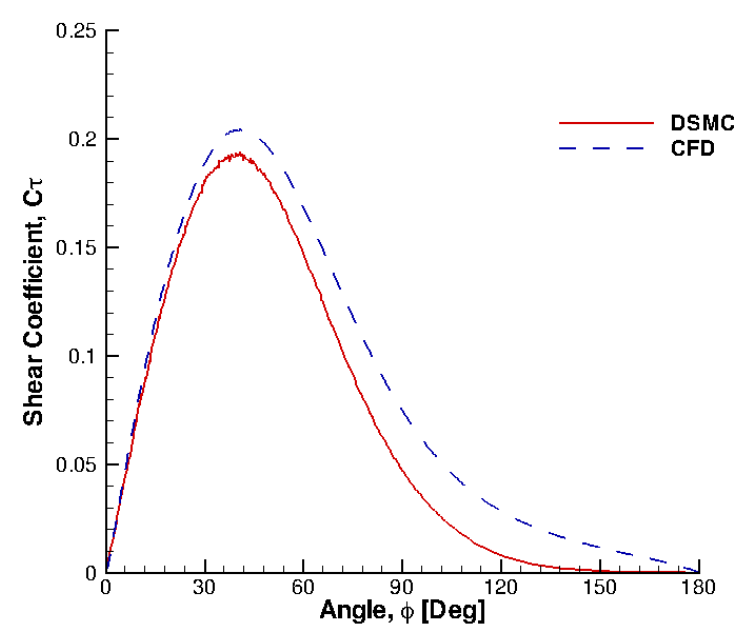

(b) Coefficient of Shear Stress

Figure 16. $K n_{\infty}=0.01$, Surface heat flux and shear stress on a sphere in a Mach 45 flow of nitrogen

Since there is no clear trend with increasing Mach number, as there is with increasing global Knudsen 
number, one can conclude that Mach number does not affect the surface properties in the same way as Knudsen number. Even though the amount of continuum breakdown has increased with increasing Mach number, the difference in the surface properties between CFD and DSMC remains relatively constant. This behavior can be better understood by looking at $K n_{G L L}$ along the stagnation streamline for the three Mach numbers, as given in Fig. 17. From this figure it is seen that the region of continuum breakdown increases with Mach number, but $K n_{G L L}$ is not significantly changed. Continuum breakdown increases because the thickness of the shock increases, that is seen in the figure by the fact that the profile of $K n_{G L L}$ widens with increasing Mach number.

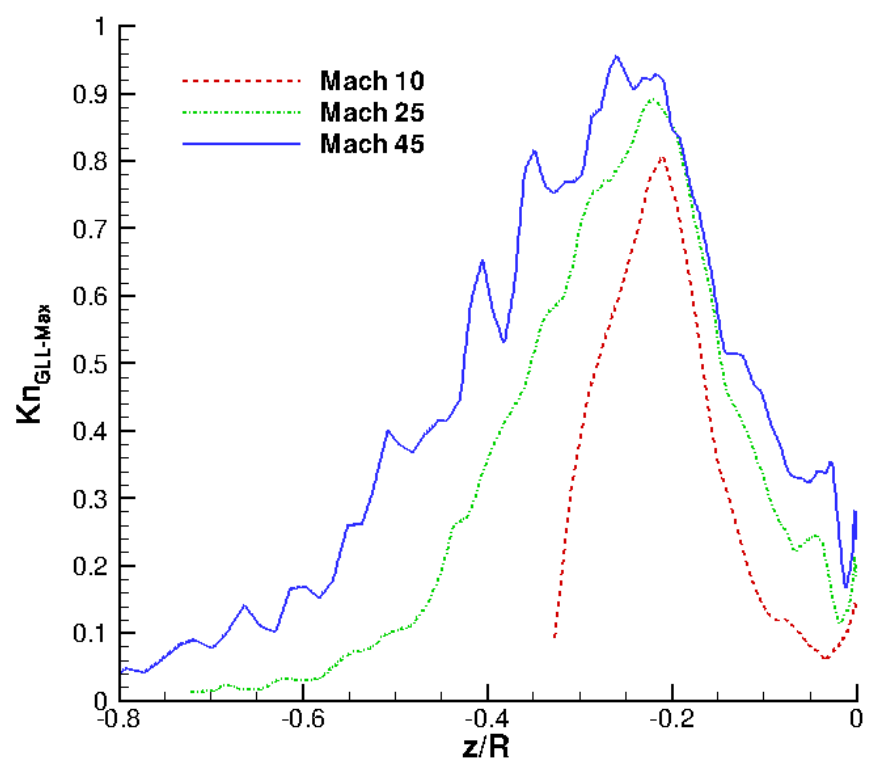

Figure 17. $K n_{G L L}$ along the stagnation stream line at a global Knudsen number of 0.01

\section{IV.D. Comparison of Sphere and Cylinder Flows}

A further part of this study compares the results of the sphere with that of the analogous cylinder case. This is performed to compare the amount of continuum breakdown and the effects on the surface properties caused by changing the geometry. Since the main focus of this study is on the sphere, only one case is employed to make a comparison to the cylinder. The case chosen for this comparison is the global Knudsen number of 0.01 case at Mach 10. The integrated drag and peak heat flux are given in Tables 6 and 7 , respectively. The tables give the values predicted by DSMC and CFD along with a percent difference between DSMC and CFD. Once again, it is assumed that the DSMC results are more accurate, so the percent difference is calculated using the DSMC result. For both the peak heat flux and the integrated drag, the axisymmetric case has much larger differences between the two methods.

Table 6. Integrated Drag [N] for DSMC and CFD for a Knudsen Number of 0.01 and a Mach number of 10

\begin{tabular}{|c|c|c|c|}
\hline & DSMC & CFD & Percent Difference \\
\hline \hline Cylinder & 33.96 & 34.14 & $0.504 \%$ \\
Sphere & 30.78 & 31.62 & $2.69 \%$ \\
\hline
\end{tabular}

From the integrated drag and the total heat flux it can be seen that running a simulation axisymmetrically leads to larger differences between DSMC and CFD. So the question becomes, how does axisymmetry affect continuum breakdown. This question can be answered by comparing the plots of $K n_{G L L}$ for the sphere 
Table 7. Peak Heating $\left[\frac{W}{m^{2}}\right]$ for DSMC and CFD for a Knudsen Number of 0.01 and a Mach number of 10

\begin{tabular}{|c|c|c|c|}
\hline & DSMC & CFD & Percent Difference \\
\hline \hline Cylinder & $3.2 \times 10^{4}$ & $3.34 \times 10^{4}$ & $4.63 \%$ \\
Sphere & $4.65 \times 10^{4}$ & $5.64 \times 10^{4}$ & $17.38 \%$ \\
\hline
\end{tabular}

and cylinder case, as presented in Fig. 18. This figure shows contours of $K n_{G L L}$ for DSMC and CFD for the cylinder and sphere cases. It is seen that the DSMC prediction of $K n_{G L L}$ has a much larger amount of continuum breakdown in the wake of the sphere than for the cylinder. It is interesting to note that the amount of breakdown in the shock is approximately the same. The CFD prediction of $K n_{G L L}$ remains nearly the same for both the sphere and the cylinder. The shock in the cylinder case is farther out than for the sphere, but this is expected due to the ability of gas to flow in all directions around the sphere thus allowing the shock to move closer to the surface.

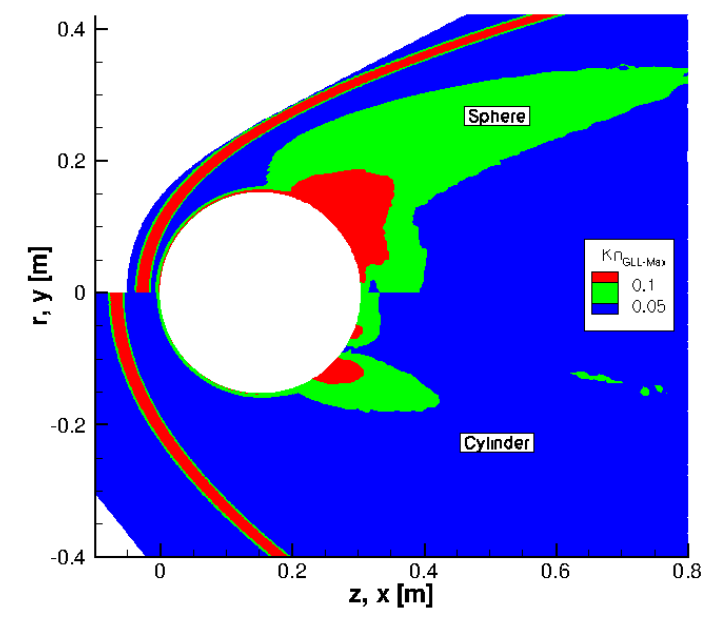

(a) DSMC

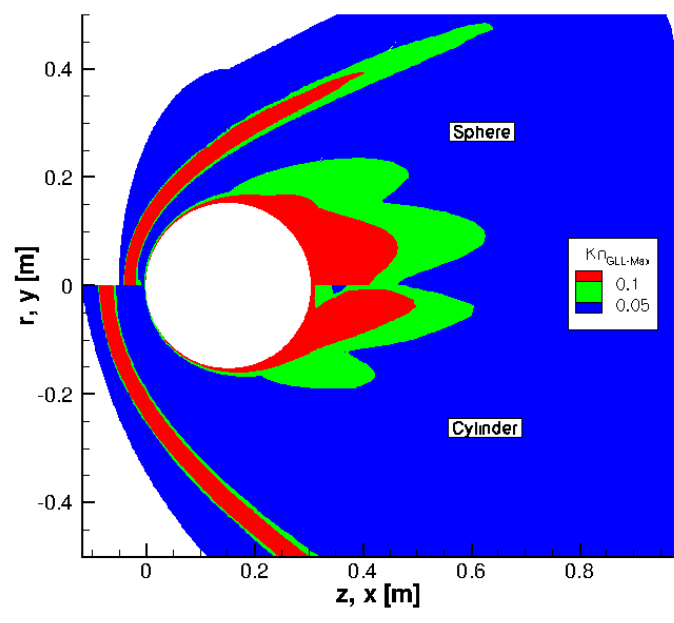

(b) $\mathrm{CFD}$

Figure 18. $K n_{\infty}=0.01, K n_{G L L}$ from DSMC and CFD in a Mach 10 flow of nitrogen over a sphere and a cylinder

Figure 19 gives the temperature and $K n_{G L L}$ profiles along the stagnation stream line for the sphere and the cylinder. From this figure it is seen that the flow is in continuum breakdown from the shock to the surface for the sphere while for the cylinder there is only breakdown in the shock and in the boundary layer. Since there is less breakdown for the cylinder case, the temperature profiles for CFD and DSMC match very well. The only difference in the temperature profiles for the cylinder, is that the shock is thicker in DSMC so the temperatures begin increasing slightly farther upstream than CFD. Also, notice that the rotational temperature predicted by CFD does not overshoot the DSMC solution as it does with the sphere. Once again this is due to less continuum breakdown between the shock and the surface of the cylinder.

\section{Conclusion}

This study investigated the effects of continuum breakdown on the surface aerothermodynamic properties (pressure, stress, heat transfer rate) of a sphere in Mach 10, 25, and 45 flows of nitrogen gas in regimes varying from continuum flow to a rarefied gas flow. The first part of this paper focused on the effects of increasing global Knudsen number on continuum breakdown and the surface properties. The peak heat flux differences increased from 5 to 60 percent while the integrated drag difference increased from 0.1 to 28 percent for global Knudsen numbers varying from 0.002 to 0.25 . It was observed that with increasing global Knudsen number, the amount of continuum breakdown increased and this increase had a large effect on the surface properties of the sphere. The second part of this study was concerned with the effects of increasing Mach 


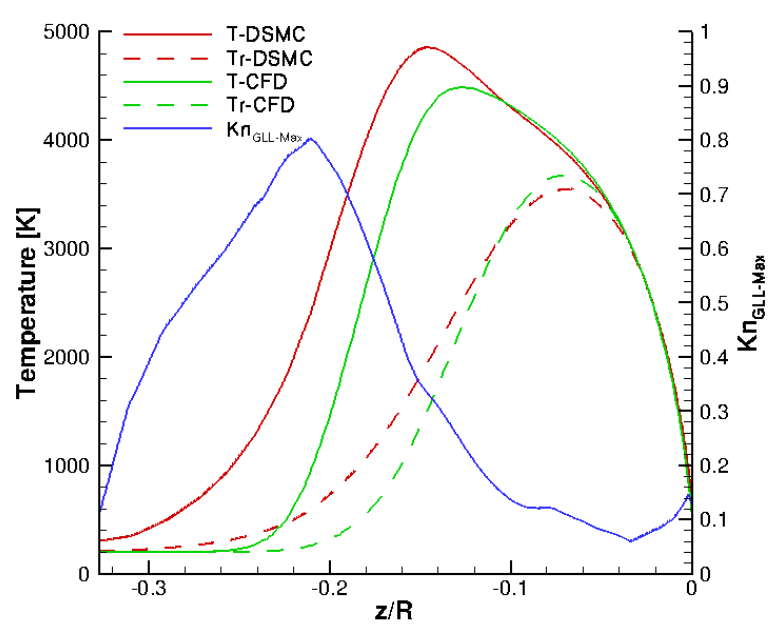

(a) Sphere

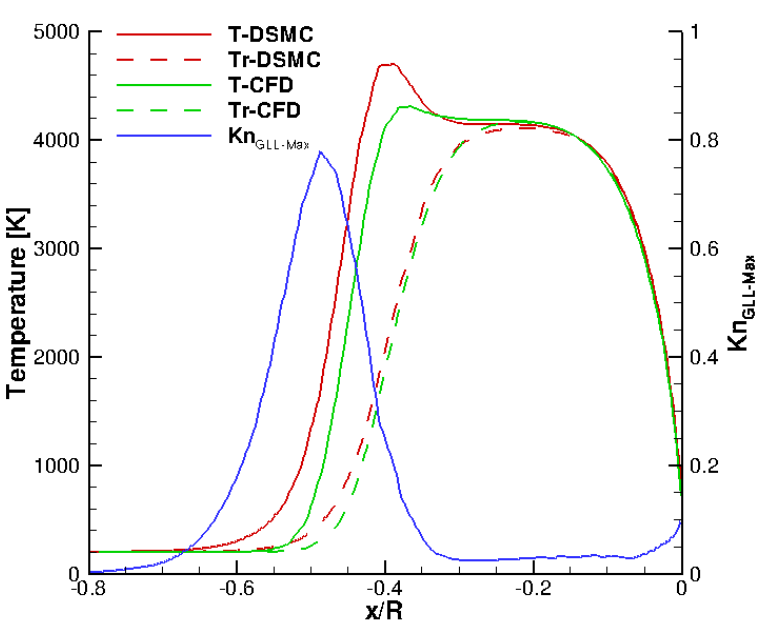

(b) Cylinder

Figure 19. $K n_{\infty}=0.01$, Temperature and $K n_{G L L}$ profiles along the stagnation stream line at Mach 10

number on continuum breakdown and the surface properties. As Mach number was increased, the amount of continuum breakdown also increased, but there were no clear effects of this increase in breakdown on the surface properties. Even though the amount of continuum breakdown increased with Mach number, the value of $K n_{G L L}$ remained relatively constant causing the differences in the surface properties between CFD and DSMC to be nearly unchanged. The last part of this study compared the results of the sphere with the analogous case of a two dimensional cylinder for a global Knudsen number of 0.01 at Mach 10. The CFD integrated drag prediction was higher by 3 percent from the DSMC prediction, while it was 0.5 percent higher for the equivalent cylinder case. The peak heat transfer predicted by CFD was higher by 17 percent, while it was 5 percent higher for the cylinder case. For DSMC the amount of continuum breakdown increased when run axisymmetrically as compared to the two dimensional case, however this trend is not observed in CFD. It is clear that continuum breakdown affects surface properties, but the effects are much less noticeable on the surface pressure than on shear stress or heat flux. From this study it has been observed that varying the global Knudsen number, Mach number and running the simulation axisymmetrically all have an effect on the amount of continuum breakdown.

\section{Future Work}

This study focused on flow in nitrogen gas. Future studies will investigate the effects of continuum break down in multi-species gases, such as including oxygen, to better simulate the effects of continuum breakdown in air. Further investigation into the effects of chemical reactions in nitrogen as well as air will be performed. It is also of interest to investigate continuum breakdown in fully three dimensional simulations of shapes more representative of blunt body re-entry vehicles. Also, slip and temperature jump boundary conditions in the CFD model will be considered, similar to Lofthouse et al. ${ }^{5}$ Including velocity slip and temperature jump boundary conditions in the CFD model will most likely improve the predicted surface properties, especially since DSMC naturally includes slip velocity and temperature jump at the surface as shown in Fig. 20.

\section{Acknowledgments}

This work is sponsored under a NASA graduate student researchers program fellowship (Grant NNX07AV91H) through NASA Ames Research Center monitored by Dr. Michael Wright. The use of supercomputing resources from the University of Michigan and NASA is essential to this investigation and is greatly appreciated. 


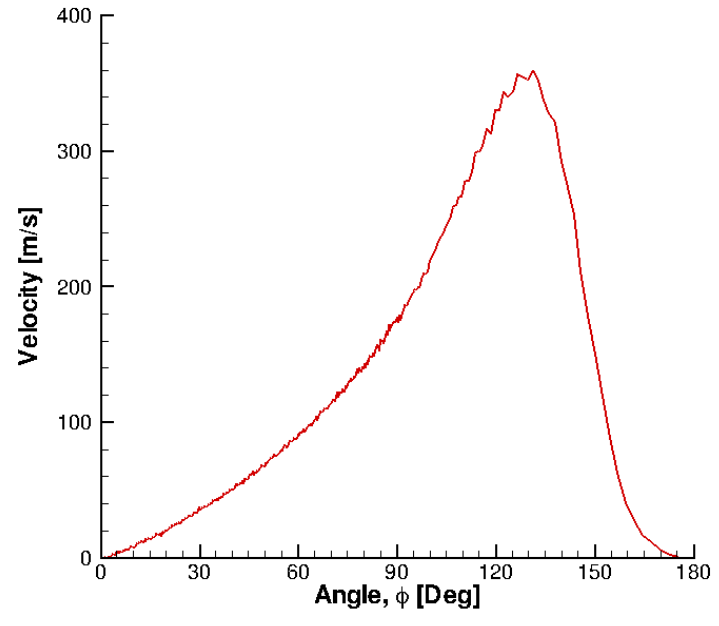

(a) Slip Velocity

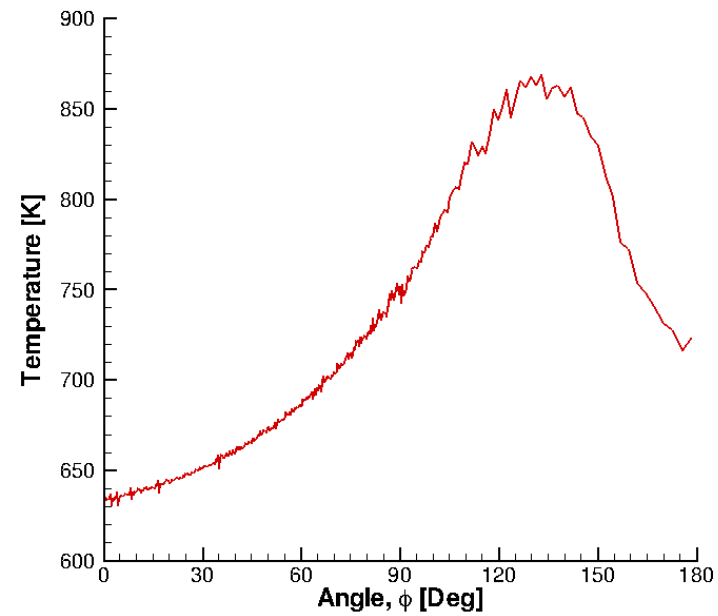

(b) Temperature Jump

Figure 20. $K n_{\infty}=0.01$, Slip Velocity and Temperature Jump from DSMC for a sphere in a Mach 10 Flow of Nitrogen

\section{References}

${ }^{1}$ Vincenti, W. G. and Kruger, C. H., Intorduction to Physical Gas Dynamics, Krieger Publishing Company, 1965.

${ }^{2}$ Bird, G. A., Molecular Gas Dynamics and the Direct Simulation of Gas Flows, Oxford University Press, 1994.

${ }^{3}$ Boyd, I. D., Chen, G., and Chandler, G., "Predicting Failure of the Continuum Fluid Equations in Transitional Hypersonic Flows," Physics of Fluids, Vol. 7, 1995, pp. 210-219.

${ }^{4}$ Lofthouse, A. J., Boyd, I. D., and Wright, M. J., "Effects of Continuum Breakdown on Hypersonic Aerothermodynamics," Physics of Fluids, Vol. 19, 2007, Article 027105.

${ }^{5}$ Lofthouse, A. J., Scalabrin, L. C., and Boyd, I. D., "Velocity Slip and Temperature Jump in Hypersonic Aerothermodynamics," Journal of Thermophysics and Heat Transfer, Vol. 22, No. 1, 2008, pp. 38-48.

${ }^{6}$ Dietrich, S. and Boyd, I. D., "Scalar and Parallel Optimized Implementation of the Direct Simulation Monte Carlo Method," Journal of Computational Physics, Vol. 126, 1996, pp. 328-342.

${ }^{7}$ Kannenberg, K. C. and Boyd, I. D., "Stategies for Efficient Particle Resolution in the Direct Simulation Monte Carlo Method," Journal of Computational Physics, Vol. 157, 2000, pp. 727-745.

${ }^{8}$ Scalabrin, L. C. and Boyd, I. D., "Development of an Unstructured Navier-Stokes Solver for Hypersonic Nonequilibrium Aerothermodynamics," American Institute of Aeronautics and Astronautics, 2005, AIAA 2005-52035, 38th AIAA Thermophysics Conference, Toronto, Canada, June.

${ }^{9}$ Scalabrin, L. C. and Boyd, I. D., "Numerical Simulation of Weakly Ionized Hypersonic Flow for Reentry Configurations," American Institute of Aeronautics and Astronautics, 2006, AIAA 2006-3773, 9th AIAA/ASME Joint Thermophysics and Heat Transfer Conference, San Francisco, CA, June.

${ }^{10}$ Schwartzentruber, T. E., Scalabrin, L. C., and Boyd, I. D., "Hybrid Particle-Continuum Simulations of Non-Equilibrium Hypersonic Blunt Body Flow Fields," American Institute of Aeronautics and Astronautics, 2006, AIAA 2006-3602, 9th AIAA/ASME Joint Thermophysics and Heat Transfer Conference, San Francisco, CA, June.

${ }^{11}$ Boyd, I. D., "Analysis of Rotational Nonequilibrium in Standing Shock Waves of Nitrogen," American Institute of Aeronautics and Astronautics, Vol. 28, 1990, pp. 1997-1999.

${ }^{12}$ Parker, J. G., "Rotational and Vibrational Relaxation in Diatomic Gases," Physics of Fluids, Vol. 2, 1959, pp. 449-462. 